\title{
A CAUTIONARY TALE: MARVELS BROWN DWARF CANDIDATE REVEALS ITSELF TO BE A VERY LONG PERIOD, HIGHLY ECCENTRIC SPECTROSCOPIC STELLAR BINARY
}

\author{
Claude E. Mack III ${ }^{1}$, Jian Ge ${ }^{2}$, Rohit Deshpande ${ }^{3,4}$, John P. Wisniewski ${ }^{5}$, Keivan G. Stassun ${ }^{1,6}$, B. Scott Gaudi ${ }^{7}$, \\ Scott W. Fleming ${ }^{2,3,4}$, Suvrath Mahadevan ${ }^{3,4}$, Nathan De LeE $^{1,2}$, Jason Eastman $^{7}$, Luan Ghezzi $^{8,9}$, \\ Jonay I. González Hernández ${ }^{10,11}$, Bruno Femenía ${ }^{10,11}$, Letícia Ferreira ${ }^{9,12}$, Gustavo Porto de Mello ${ }^{9,12}$, \\ Justin R. Crepp ${ }^{13}$, Daniel Mata Sánchez ${ }^{10,11}$, Eric Agol ${ }^{14}$, Thomas G. Beatty ${ }^{7}$, Dmitry Bizyaev ${ }^{15}$, \\ Howard Brewington ${ }^{15}$, Phillip A. Cargile ${ }^{1}$, Luiz N. Da Costa ${ }^{8,9}$, Massimiliano Esposito ${ }^{10,11}$, Garket Ebelke ${ }^{15}$, \\ Leslie Hebb $^{1,14}$, Peng Jiang ${ }^{2}$, Stephen R. Kane ${ }^{16}$, Brian LeE ${ }^{2}$, Marcio A. G. Maia ${ }^{8,9}$, Elena Malanushenko ${ }^{15}$, \\ Victor Malanushenko ${ }^{15}$, Daniel Oravetz ${ }^{15}$, Martin Paegert ${ }^{1}$, Kaike Pan ${ }^{15}$, Carlos Allende Prieto ${ }^{10,11}$, \\ Joshua Pepper ${ }^{1}$, Rafael Rebolo ${ }^{10,11}$, Arpita Roy ${ }^{3}$, Basílio X. Santiago ${ }^{9,17}$, Donald P. Schneider ${ }^{3,4}$, Audrey Simmons ${ }^{15}$, \\ Robert J. Siverd ${ }^{1}$, Stephanie Snedden $^{15}$, and Benjamin M. Tofflemire ${ }^{18}$ \\ ${ }^{1}$ Department of Physics and Astronomy, Vanderbilt University, Nashville, TN 37235, USA; claude.e.mack@ vanderbilt.edu \\ ${ }^{2}$ Department of Astronomy, University of Florida, 211 Bryant Space Science Center, Gainesville, FL, 32611-2055, USA \\ ${ }^{3}$ Department of Astronomy and Astrophysics, The Pennsylvania State University, 525 Davey Laboratory, University Park, PA 16802, USA \\ ${ }^{4}$ Center for Exoplanets and Habitable Worlds, Pennsylvania State University, University Park, PA 16802, USA \\ ${ }^{5}$ Homer L Dodge Department of Physics \& Astronomy, University of Oklahoma, 440 W Brooks St, Norman, OK 73019, USA \\ ${ }^{6}$ Department of Physics, Fisk University, Nashville, TN, USA \\ ${ }^{7}$ Department of Astronomy, The Ohio State University, 140 West 18th Avenue, Columbus, OH 43210, USA \\ ${ }^{8}$ Observatório Nacional, Rua Gal. José Cristino 77, Rio de Janeiro, RJ 20921-400, Brazil \\ ${ }^{9}$ Laboratório Interinstitucional de e-Astronomia-LIneA, Rua Gal. José Cristino 77, Rio de Janeiro, RJ 20921-400, Brazil \\ ${ }^{10}$ Instituto de Astrofísica de Canarias (IAC), E-38205 La Laguna, Tenerife, Spain \\ ${ }^{11}$ Departamento de Astrofísica, Universidad de La Laguna, E-38206 La Laguna, Tenerife, Spain \\ ${ }^{12}$ Observatório do Valongo, Universidade Federal do Rio de Janeiro, Ladeira do Pedro Antonio 43, Rio de Janeiro, RJ 20080-090, Brazil \\ ${ }^{13}$ Department of Physics, University of Notre Dame, 225 Nieuwland Science Hall, Notre Dame, IN 46556, USA \\ ${ }^{14}$ Astronomy Department, University of Washington, Box 351580, Seattle, WA 98195, USA \\ ${ }^{15}$ Apache Point Observatory, P.O. Box 59, Sunspot, NM 88349-0059, USA \\ ${ }^{16}$ NASA Exoplanet Science Institute, Caltech, MS 100-22, 770 South Wilson Avenue, Pasadena, CA 91125, USA \\ ${ }^{17}$ Instituto de Física, UFRGS, Caixa Postal 15051, Porto Alegre, RS 91501-970, Brazil \\ ${ }^{18}$ Astronomy Department, University of Wisconsin-Madison, 475 N Charter St, Madison, WI 53706, USA \\ Received 2013 January 1; accepted 2013 March 14; published 2013 April 9
}

\begin{abstract}
We report the discovery of a highly eccentric, double-lined spectroscopic binary star system (TYC 3010-1494-1), comprising two solar-type stars that we had initially identified as a single star with a brown dwarf companion. At the moderate resolving power of the MARVELS spectrograph and the spectrographs used for subsequent radialvelocity $(\mathrm{RV})$ measurements $(R \lesssim 30,000)$, this particular stellar binary mimics a single-lined binary with an RV signal that would be induced by a brown dwarf companion $\left(M \sin i \sim 50 M_{\mathrm{Jup}}\right)$ to a solar-type primary. At least three properties of this system allow it to masquerade as a single star with a very-low-mass companion: its large eccentricity $(e \sim 0.8)$, its relatively long period $(P \sim 238$ days $)$, and the approximately perpendicular orientation of the semi-major axis with respect to the line of sight $\left(\omega \sim 189^{\circ}\right)$. As a result of these properties, for $\sim 95 \%$ of the orbit the two sets of stellar spectral lines are completely blended, and the RV measurements based on centroiding on the apparently single-lined spectrum is very well fit by an orbit solution indicative of a brown dwarf companion on a more circular orbit $(e \sim 0.3)$. Only during the $\sim 5 \%$ of the orbit near periastron passage does the true, double-lined nature and large RV amplitude of $\sim 15 \mathrm{~km} \mathrm{~s}^{-1}$ reveal itself. The discovery of this binary system is an important lesson for RV surveys searching for substellar companions; at a given resolution and observing cadence, a survey will be susceptible to these kinds of astrophysical false positives for a range of orbital parameters. Finally, for surveys like MARVELS that lack the resolution for a useful line bisector analysis, it is imperative to monitor the peak of the cross-correlation function for suspicious changes in width or shape, so that such false positives can be flagged during the candidate vetting process.
\end{abstract}

Key words: binaries: spectroscopic - brown dwarfs - stars: individual (TYC 3010-1494-1)

Online-only material: color figures

\section{INTRODUCTION}

As a part of the third phase of the Sloan Digital Sky Survey (SDSS-III; Eisenstein et al. 2011), the MARVELS (Multi-object APO Radial Velocity Exoplanet Large-area Survey) project is searching for substellar companions by monitoring the radial velocities (RVs) of 3330 FGK stars (Ge et al. 2008, 2009; Ge \& Eisenstein 2009). This sample size is large enough for the project to find relatively rare objects, such as brown dwarf (BD) companions to solar-type stars. The paucity of observed BD companions to solar-type stars with separations of $\lesssim 5 \mathrm{AU}$ is typically referred to as the BD desert (Marcy \& Butler 2000). Since the size of the MARVELS sample allows us to begin to quantify how arid the BD desert may be, any MARVELS discovery of a BD in the desert (or lack thereof) is a step toward increasing our understanding of BD formation.

In addition to its large homogeneous target sample, MARVELS differs from other surveys for substellar companions 


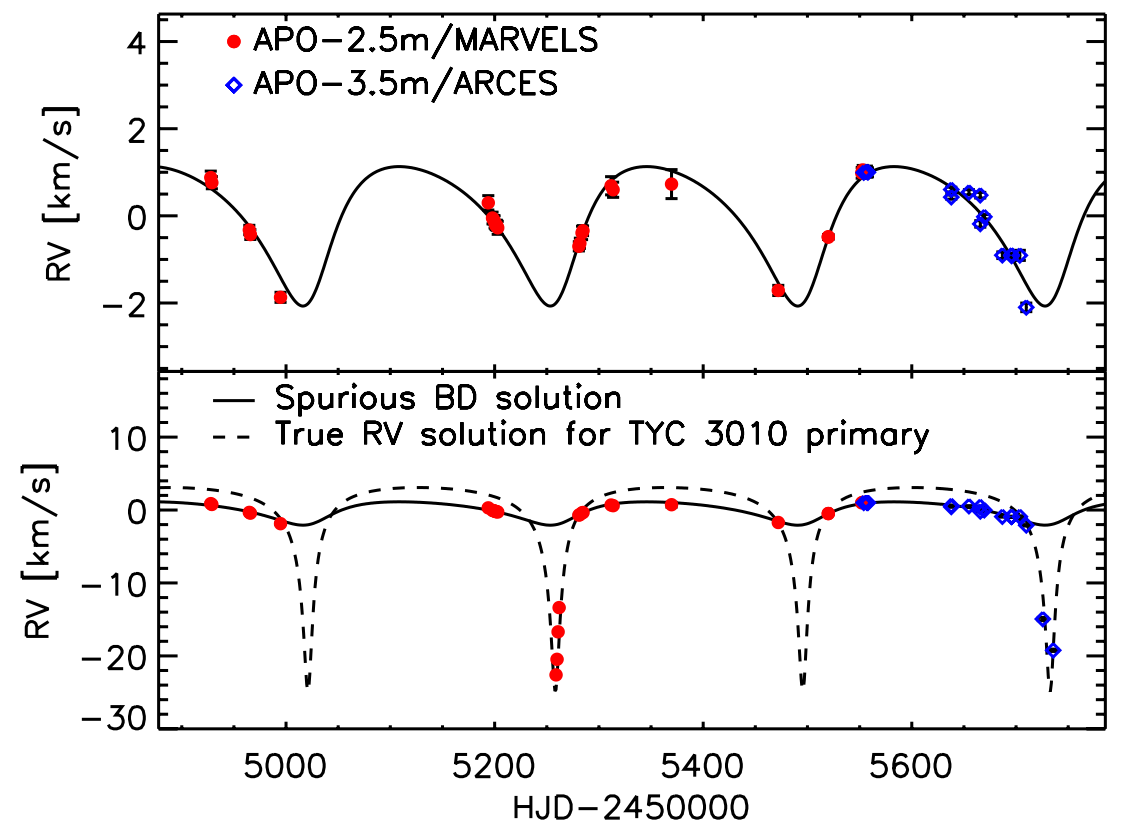

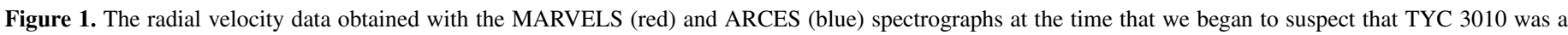

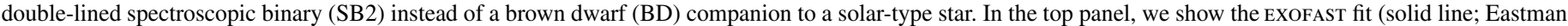

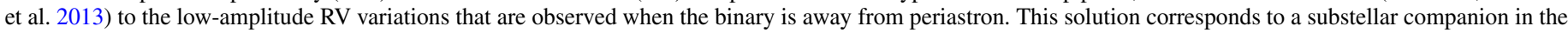

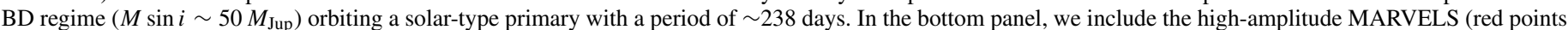

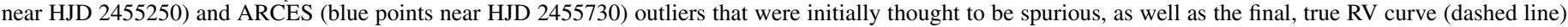

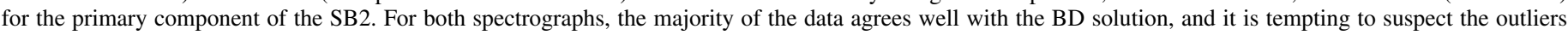

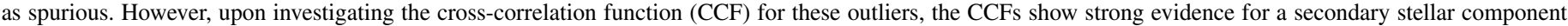

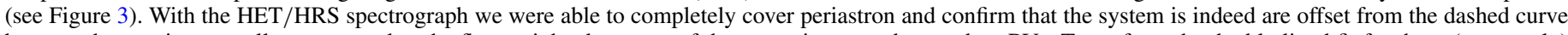

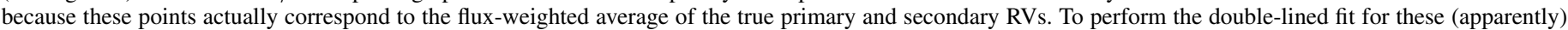
single-lined epochs, we first disentangled the primary and secondary components as described in Section 3.2.1.

(A color version of this figure is available in the online journal.)

in two key ways. First, the project employs a dispersed fixeddelay interferometer (DFDI; Ge 2002; Ge et al. 2002, 2006; Erskine 2003; van Eyken et al. 2010; Wang et al. 2011). Second, it uses a multi-object spectrograph to observe 60 stars simultaneously (Ge et al. 2009). The DFDI prototype instrument was used to discover the first extrasolar planet around HD 102195 in 2006 with this new RV method (Ge et al. 2006). The MARVELS DFDI technique combines an interferometer with a medium resolution spectrograph $(R \sim 12,000)$ in order to obtain a precision of $\sim 100 \mathrm{~m} \mathrm{~s}^{-1}$. Given its RV precision and survey design to monitor each target with at least $24 \mathrm{RV}$ measurements over at least $1 \mathrm{yr}$, MARVELS is sensitive to BD and low-mass stellar companions with periods ranging from a few days to hundreds of days. Nonetheless, certain specific types of astrophysical false positives can mimic substellar companions unless additional vetting is performed. This paper describes just such a case, TYC-3010-1494-1 (hereafter TYC 3010), a stellar binary that initially appeared as a single star with a substellar companion and that, through a confluence of orbital parameters, continued to masquerade as such despite a disconcertingly extensive amount of observation and analysis.

When we began analysis of TYC 3010, MARVELS and its pilot project had already detected two BD candidates orbiting late F stars in the BD desert (Fleming et al. 2010; Lee et al. 2011, at present, we have three more candidates in the desert: Ma et al. 2013; Jiang et al. 2013; De Lee et al. 2013). The MARVELS discovery data indicated that TYC 3010 possessed a substellar companion with a minimum mass of $\sim 50 M_{\text {Jup }}$ and that it was on a $\sim 238$ day moderately eccentric orbit with an RV amplitude of $\sim 1.5 \mathrm{~km} \mathrm{~s}^{-1}$ (see the top panel of Figure 1). However, given the cadence of MARVELS and the period of the orbit, there were significant gaps in the phase coverage and additional observations with a different spectrograph were required to constrain the RV solution. Initially, the follow-up data remained fully consistent with the $\mathrm{BD}$ companion scenario. However, during the course of the program, we found two RV points that were shifted by $\sim 20 \mathrm{~km} \mathrm{~s}^{-1}$ with respect to most of our data; while investigating the source of these anomalous points, we realized that a few similar points had been rejected from our MARVELS discovery data by the team's outlier rejection procedures (see bottom panel of Figure 1). Examining the cross-correlation function (CCF) of the anomalous RV points (in both the discovery and subsequent data) revealed evidence that there were two components in the CCF, which suggested that the companion to the primary was most likely a stellarmass secondary. Finally, including the initially flagged outlier measurements and disentangling the RV measurements of the two components, the system was found to be a nearly equal-mass stellar binary $(q \sim 0.88)$ on a highly eccentric orbit $(e \sim 0.8)$. Evidently, for a system like TYC 3010, it is possible to clip just a few measurements and obtain an apparently reasonable solution that is convincing but completely incorrect.

As large scale RV and transit surveys for exoplanets become more common, it is increasingly inevitable that any and all forms of astrophysical false positives, despite their rarity, will be found. Indeed, the first BD candidate discovered by the MARVELS project, MARVELS-1 (Lee et al. 2011), appeared to exhibit evidence for an additional planet-mass companion, but turned out instead to likely be a quadruple system, comprising four stars with no detected BD or planetary-mass companion (Wright et al. 2013). Akin to TYC 3010, MARVELS-1 is a double-lined spectroscopic binary; the stars have relative 
RVs which are sufficiently low that they are always blended, even at the resolution of Hobby-Eberly Telescope (HET; $R \sim$ 60,000 mode). Thus, with both MARVELS-1 and TYC 3010, we actually measure a flux-weighted mean of two sets of stellar spectral lines. This flux-weighted mean exhibits a suppressed velocity shift that mimics a single-lined binary with a BD secondary. Both systems possess geometries that allow them to masquerade as less massive systems: MARVELS- 1 is nearly face-on, which leads to low projected velocities, while TYC 3010 is on a highly elliptical orbit with a semi-major axis oriented nearly perpendicular to our line of sight.

Similarly, Mandushev et al. (2005) describe what at first appeared to be a transiting BD companion to an $\mathrm{F}$ star from the TRES transit survey, but turned out instead to be an F star blended with a $\mathrm{G}+\mathrm{M}$ stellar eclipsing binary. The system that we describe here follows these unfortunate examples, and is similarly pernicious.

In the following sections, we present our analysis as a kind of cautionary tale for other RV surveys to avoid similar false positives. In Section 2, we describe the spectroscopic and photometric data obtained for TYC 3010. In Section 3, we discuss in detail the nature of the evidence that led us to conclude that TYC 3010 was an eccentric stellar binary instead of a BD companion to a solar-type star. We also present the properties we derived for both components of the spectroscopic binary. In Section 4, we discuss the circumstances that allowed this false positive to masquerade for so long and through several vetting steps as a compelling detection of a substellar companion, and we describe methods that the MARVELS team and other RV surveys can use to recognize this kind of astrophysical false positive in the future. Finally, in Section 5, we conclude with a summary of the main results.

\section{OBSERVATIONS AND DATA PROCESSING}

We obtained a total of $65 \mathrm{RV}$ measurements from the Sloan $2.5 \mathrm{~m}$, the APO $3.5 \mathrm{~m}$, and the HET $9.2 \mathrm{~m}$ telescopes. We will briefly summarize the characteristics of the data from all three telescopes. For more details of the analysis, please see Fleming et al. (2010), Lee et al. (2011), and Wisniewski et al. (2012).

\subsection{SDSS-III MARVELS Discovery RV Data}

A total of 28 spectra (see Table 1) of TYC 3010 were obtained with the Sloan $2.5 \mathrm{~m}$ telescope (Gunn et al. 2006) at Apache Point Observatory (APO). The multi-fiber MARVELS spectrograph (Ge et al. 2009) can simultaneously measure the RVs of 60 stars during each telescope pointing. Both beams of the interferometer are imaged onto the detector, so each 50 minute observation results in two fringed spectra in the wavelength range of $\sim 500-570 \mathrm{~nm}$ with a resolving power of $R \sim 12,000$. The MARVELS interferometer delay calibrations are described in Wang et al. (2012a, 2012b). For more details on how the data were reduced and analyzed to yield RVs, see Lee et al. (2011).

As described below, it proved essential to examine the $\mathrm{CCFs}$ of the individual spectra. However, performing a crosscorrelation on a DFDI spectrum requires a few steps beyond what one performs for a typical slit or cross-dispersed echelle spectrograph. In both cases the images are reduced using standard techniques (bias subtraction, trace correction, flat fielding etc.) Once a fully processed two-dimensional spectrum has been extracted, there is a divergence in the techniques. In the case of a normal spectrum, one merely sums the flux in the
Table 1

Observed Heliocentric Single-lined Radial Velocities for TYC 3010

\begin{tabular}{|c|c|c|c|}
\hline HJD & Instrument $^{\mathrm{a}}$ & $\begin{array}{c}\mathrm{RV} \\
\left(\mathrm{km} \mathrm{s}^{-1}\right)\end{array}$ & $\begin{array}{c}\sigma_{\mathrm{RV}} \\
\left(\mathrm{km} \mathrm{s}^{-1}\right)\end{array}$ \\
\hline 2454927.82470 & M & 62.681 & 0.148 \\
\hline 2454928.85061 & M & 62.564 & 0.139 \\
\hline 2454964.76792 & $\mathrm{M}$ & 61.479 & 0.108 \\
\hline 2454965.77714 & M & 61.374 & 0.113 \\
\hline 2454994.69536 & M & 59.933 & 0.115 \\
\hline 2455193.91250 & $\mathrm{M}$ & 62.102 & 0.165 \\
\hline 2455197.96727 & M & 61.753 & 0.134 \\
\hline 2455198.94828 & M & 61.714 & 0.095 \\
\hline 2455199.96552 & M & 61.664 & 0.139 \\
\hline 2455200.98947 & M & 61.585 & 0.097 \\
\hline 2455201.97760 & M & 61.587 & 0.116 \\
\hline 2455202.99063 & $\mathrm{M}$ & 61.528 & 0.149 \\
\hline 2455258.88272 & M & 39.192 & 0.091 \\
\hline 2455259.83118 & M & 41.327 & 0.092 \\
\hline 2455260.82412 & $\mathrm{M}$ & 45.097 & 0.145 \\
\hline 2455261.82050 & M & 48.416 & 0.096 \\
\hline 2455280.77587 & M & 61.103 & 0.105 \\
\hline 2455280.76844 & $\mathrm{M}$ & 61.174 & 0.117 \\
\hline 2455283.81484 & M & 61.411 & 0.154 \\
\hline 2455284.75054 & M & 61.461 & 0.112 \\
\hline 2455311.68421 & $\mathrm{M}$ & 62.493 & 0.209 \\
\hline 2455313.62591 & M & 62.402 & 0.174 \\
\hline 2455369.64423 & M & 62.531 & 0.333 \\
\hline 2455551.99403 & M & 62.788 & 0.120 \\
\hline 2455552.98222 & M & 62.856 & 0.104 \\
\hline 2455553.98561 & M & 62.795 & 0.121 \\
\hline 2455556.97163 & M & 62.821 & 0.123 \\
\hline 2455557.97465 & M & 62.801 & 0.104 \\
\hline 2455471.98302 & A & 60.138 & 0.116 \\
\hline 2455519.95995 & A & 61.359 & 0.052 \\
\hline 2455519.98157 & A & 61.371 & 0.051 \\
\hline 2455637.88366 & A & 62.452 & 0.055 \\
\hline 2455637.92209 & A & 62.278 & 0.048 \\
\hline 2455654.83350 & A & 62.390 & 0.059 \\
\hline 2455665.65219 & A & 62.323 & 0.065 \\
\hline 2455665.69165 & A & 61.664 & 0.075 \\
\hline 2455669.60113 & A & 61.827 & 0.052 \\
\hline 2455686.82409 & A & 60.946 & 0.076 \\
\hline 2455695.66512 & A & 60.949 & 0.039 \\
\hline 2455695.70529 & A & 60.931 & 0.053 \\
\hline 2455703.61994 & A & 60.942 & 0.116 \\
\hline 2455709.77767 & A & 59.749 & 0.098 \\
\hline 2455903.90846 & $\mathrm{H}$ & 62.448 & 0.051 \\
\hline 2455917.87269 & $\mathrm{H}$ & 62.237 & 0.060 \\
\hline 2455928.84083 & $\mathrm{H}$ & 61.759 & 0.046 \\
\hline 2455940.80855 & $\mathrm{H}$ & 61.122 & 0.058 \\
\hline 2455946.80490 & $\mathrm{H}$ & 60.285 & 0.055 \\
\hline 2455950.80134 & $\mathrm{H}$ & 59.539 & 0.045 \\
\hline 2455953.82447 & A & 58.385 & 0.049 \\
\hline 2455954.00566 & $\mathrm{H}$ & 58.467 & 0.050 \\
\hline
\end{tabular}

Notes. The ARCES and HRS RV values were measured as absolute heliocentric RVs, while the MARVELS discovery data were measured on a relative instrumental scale; the MARVELS RVs have been offset to the same (heliocentric) scale as the ARCES and HRS measurements.

${ }^{a}$ Instruments: MARVELS (M), ARCES (A), and HRS (H) spectrographs.

slit (channel) direction to produce a one-dimensional spectrum. This approach is not possible in the DFDI technique because the fringing pattern will introduce false fluctuations in total flux if one just sums in the slit direction. These fluctuations will be a function of the phase of the fringe pattern in each pixel channel. To correct for this effect, a sinusoidal function of the form $A \sin (w x+b)+c$ is fit to each pixel column. For the 
Table 2

Observed Heliocentric Double-lined Radial Velocities for TYC 3010

\begin{tabular}{lccccc}
\hline \hline $\mathrm{HJD}$ & Instrument $^{\mathrm{a}}$ & $\begin{array}{c}\mathrm{RV}_{\text {primary }} \\
\left(\mathrm{km} \mathrm{s}^{-1}\right)\end{array}$ & $\begin{array}{c}\sigma_{\mathrm{RV}_{\text {primary }}} \\
\left(\mathrm{km} \mathrm{s}^{-1}\right)\end{array}$ & $\begin{array}{c}\mathrm{RV}_{\text {secondary }} \\
\left(\mathrm{km} \mathrm{s}^{-1}\right)\end{array}$ & $\begin{array}{c}\sigma_{\mathrm{RV}} \\
\left(\mathrm{km} \mathrm{s}^{-1}\right)\end{array}$ \\
\hline 2455725.68377 & $\mathrm{~A}$ & 46.012 & 0.167 & 75.222 & 0.257 \\
2455735.62781 & $\mathrm{~A}$ & 43.197 & 0.251 & 81.056 & 0.175 \\
2455956.76037 & $\mathrm{H}$ & 53.788 & 0.030 & 69.104 & 0.063 \\
2455959.78075 & $\mathrm{H}$ & 51.409 & 0.025 & 71.807 & 0.055 \\
2455964.75592 & $\mathrm{H}$ & 44.163 & 0.026 & 80.066 & 0.055 \\
2455964.83117 & $\mathrm{H}$ & 44.884 & 0.071 & 79.700 & 0.372 \\
2455967.75334 & $\mathrm{H}$ & 36.755 & 0.030 & 88.389 & 0.062 \\
2455967.82824 & $\mathrm{~A}$ & 37.684 & 0.076 & 88.651 & 0.471 \\
2455968.74640 & $\mathrm{H}$ & 34.456 & 0.025 & 90.966 & 0.054 \\
2455971.73989 & $\mathrm{H}$ & 36.359 & 0.029 & 88.685 & 0.060 \\
2455972.97350 & $\mathrm{H}$ & 40.368 & 0.025 & 84.354 & 0.054 \\
2455976.73787 & $\mathrm{H}$ & 50.072 & 0.024 & 73.253 & 0.051 \\
2455977.71541 & $\mathrm{H}$ & 51.788 & 0.028 & 71.580 & 0.058 \\
2455978.71767 & $\mathrm{H}$ & 52.990 & 0.026 & 69.840 & 0.056 \\
2455979.71599 & $\mathrm{H}$ & 54.160 & 0.029 & 68.450 & 0.062 \\
\hline
\end{tabular}

Note. ${ }^{a}$ Instruments: ARCES (A) and HRS (H) spectrographs.

purposes of cross-correlation the only term of interest is $c$, or the mean flux in each channel. A one-dimensional spectrum is then constructed using the $c$ term in each channel. From this point forward the $\mathrm{CCF}$ is determined using standard techniques.

\subsection{APO $3.5 \mathrm{~m} / A R C E S$ RV Data}

A total of $19 \mathrm{RV}$ observations were taken with the APO $3.5 \mathrm{~m}$ telescope using the ARC Echelle Spectrograph (ARCES; Wang et al. 2003). This spectrograph operates in the optical regime from $\sim 3600-10000 \AA$ with a resolving power of $R \sim$ 31,500. The first set of observations were taken from 2010 October to 2011 June. The second set of observations, which were undertaken with the goal of increasing phase coverage of periastron, were obtained during 2012 January-February. As shown in Tables 1 and 2, there were 15 ARCES points observed outside of periastron, and 4 points during periastron (The first two of these periastron points are where we initially resolved both the primary and secondary spectral lines - see bottom panel of Figures 1, 2, and 3-and began to suspect that the system might be a double-lined spectroscopic binary).

To achieve high-accuracy RV measurements with the echelle spectrograph, we obtained a Thorium-Argon (ThAr) exposure after every science exposure. In order to place TYC 3010 on an absolute RV scale, we also frequently bracketed our observations of TYC 3010 with observations of the RV standard HD 102158, which has an absolute RV of $28.122 \mathrm{~km} \mathrm{~s}^{-1}$ (Crifo et al. 2010; Nidever et al. 2002). From the standard deviation of the $13 \mathrm{RV}$ measurements we obtained for HD 102158 (see Table 3), we were able to determine that the ARCES spectrograph possesses an RV stability of $\sim 0.5 \mathrm{~km} \mathrm{~s}^{-1}$.

Two of the ARCES spectra were taken with longer exposure times in order to achieve a high signal-to-noise ratio $(\mathrm{S} / \mathrm{N})$ for deriving the fundamental stellar parameters (see Section 3.2.2). These two spectra were taken with an exposure time of $200 \mathrm{~s}$ and with the default slit setting described in Wisniewski et al. (2012). The data were reduced with IRAF, and after barycentric corrections and continuum normalization, the two spectra were combined to produce a final spectrum with an $\mathrm{S} / \mathrm{N}$ of $\sim 170$ per resolution element at $\sim 6500 \AA$. However, once we realized that TYC 3010 was a double-lined spectroscopic binary, we
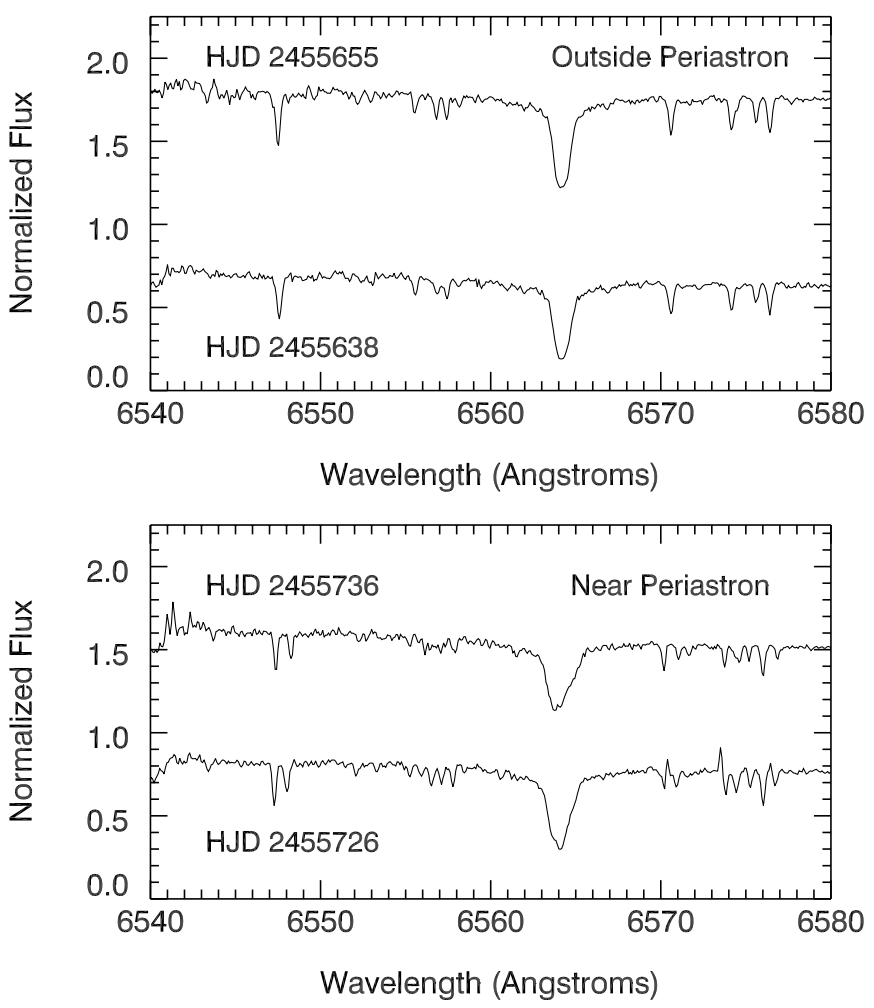

Figure 2. Top: Outside periastron the combined spectrum appears convincingly single-lined. Bottom: Near periastron the spectrum is resolved into its double-lined components (with the ARCES and HRS spectrographs, but not MARVELS). We used the double-lined spectrum with highest $\mathrm{S} / \mathrm{N}$ when we were deriving the properties of the two stars via spectral characterization.

re-derived the spectroscopic parameters with a double-lined spectrum obtained near periastron, as described in Section 3.2.2.

\subsection{HET/HRS RV Data}

Upon realizing the eccentric binary-star nature of the object from the APO $3.5 \mathrm{~m}$ data, observations where initiated using the 9.2 m HET (Ramsey et al. 1998) using the High Resolution Spectrograph (HRS; Tull 1998) at a resolving power of $R \sim 30,000$ using a 2 arcsec optical fiber. A total of 


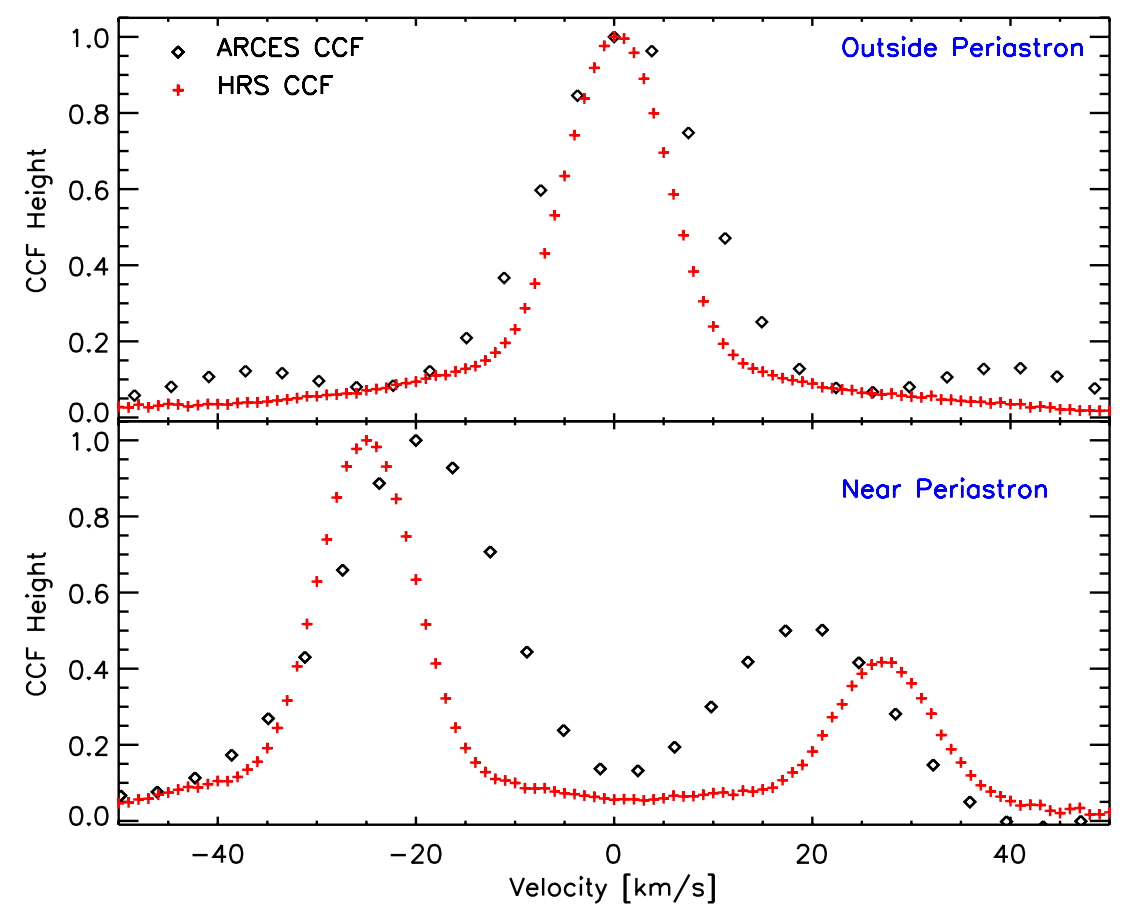

Figure 3. Example CCFs obtained with the ARCES and HRS spectrographs from similar (but different) phases outside of periastron (top panel) and during periastron (bottom panel). Since most of the data were obtained outside of periastron, most of the RV points correspond to single-peak CCFs. However, for data from near periastron, the ARCES and HRS spectrographs are able to resolve two peaks. The secondary peak is comparable in height to the primary peak, which led us to suspect that TYC 3010 is an eccentric spectroscopic binary with the semi-major axis aligned perpendicular to the line of sight (see Figure 8). With this configuration, we would only resolve two peaks in the CCF if we happen to catch the pair of stars as they briefly pass through periastron. To confirm this interpretation, we fully observed periastron with HET/HRS, which allowed us to completely constrain the orbit (see Figure 7).

(A color version of this figure is available in the online journal.)

Table 3

Observed Heliocentric Radial Velocities for the RV Standard HD 102158

\begin{tabular}{lcc}
\hline \hline HJD & $\begin{array}{c}\mathrm{RV} \\
\left(\mathrm{km} \mathrm{s}^{-1}\right)\end{array}$ & $\begin{array}{c}\sigma_{\mathrm{RV}} \\
\left(\mathrm{km} \mathrm{s}^{-1}\right)\end{array}$ \\
\hline 2455654.81577 & 28.734 & 0.052 \\
2455665.67180 & 28.050 & 0.034 \\
2455665.71479 & 28.169 & 0.043 \\
2455669.58461 & 27.736 & 0.045 \\
2455686.80825 & 27.470 & 0.054 \\
2455695.68591 & 28.030 & 0.038 \\
2455695.72570 & 28.090 & 0.034 \\
2455703.60341 & 28.244 & 0.039 \\
2455709.76151 & 27.476 & 0.030 \\
2455725.66705 & 26.692 & 0.123 \\
2455735.61237 & 28.126 & 0.109 \\
2455964.81467 & 28.093 & 0.058 \\
2455967.81369 & 27.728 & 0.050 \\
\hline
\end{tabular}

18 observations were obtained to completely cover periastron, and thereby fully constrain the orbit. The queue-scheduled observing mode of the HET (Shetrone et al. 2007) is extremely well suited for investigating objects that require monitoring over a long timespan, as well as targeted observations near periastron passage. For wavelength calibration, ThAr images were obtained immediately before and after the science exposure to aid in calibrating any possible instrument drift. The data were reduced and wavelength calibrated using custom optimal extraction scripts written in IDL. RVs were measured using two different techniques, which we describe below. The HET observations clearly resolve the orbit for TYC 3010, and constrain the eccentricity to a value of $e \sim 0.8$ (see Section 3.2 ).

\subsubsection{CCF Mask}

RVs were measured using a cross-correlation mask derived from National Solar Observatory Fourier transform spectroscopic solar data (Lytle 1993), and a technique similar to that described by Baranne et al. (1996). The resultant CCF encodes information from the $\sim 400-600 \mathrm{~nm}$ region, and we elected not to use redder wavelengths due to issues with telluric contamination. Figure 3 shows the resulting CCF for an epoch during periastron and one outside of periastron; as is the case for the ARCES data, during periastron the primary and secondary peaks are clearly visible in the HET CCFs, but outside of periastron only a single peak is resolved. The centroid of the CCF peak is determined by fitting a Gaussian.

This technique has been used successfully for isolated stars to derive precise RVs by the teams using fiber-fed high resolution spectrographs (e.g., HARPS, SOPHIE, ELODIE, CORALIE; Pepe et al. 2000; Bouchy 2006; Baranne et al. 1996; Queloz et al. 2000), since PSF stability is an important component of deriving precise RVs with this technique. Any mismatch between the CCF and the simple Gaussian model is absorbed as a zero-point offset in the derived RVs as long as the PSF is stable (resulting in a stable CCF shape). The HET/HRS spectrograph is also fiber-fed, enabling this technique to also be applied to binary stars. This method is computationally efficient, and also does not require that the spectra be normalized, resulting in a quick turn around in determining RVs once the data are in hand. The RVs derived enabled us to plan and obtain observations as soon as the peaks began to separate on the approach to peri-passage. Table 1 shows the HET RVs obtained with this technique for those epochs where the CCF appears as a single peak. 


\subsubsection{TODCOR}

While the CCF Mask technique described above works quite well, it does not yield the best RVs possible for spectra with two CCF peaks since only one mask (G2 spectral type) was used in determining peak positions. Once all the data were in hand, we were able to apply the two-dimensional cross-correlation algorithm, TODCOR (Zucker \& Mazeh 1994). TODCOR can simultaneously cross-correlate two stellar templates against a blended target stellar spectrum to disentangle the stellar RVs of the components as well as derive a flux ratio. We used TODCOR along with HRS observations of HD 161237 (G5V) and HD 198596 (K0V) as templates to measure the RVs of TYC 3010. The HRS spectrum was divided into different bandpasses, and each bandpass was solved independently following Zucker (2003) and the resulting cross-correlation surface combined with a maximum likelihood analysis. Further details on our implementation of the TODCOR algorithm, as well as details of our custom HRS spectral extraction pipeline, can be found in Bender et al. (2012).

Table 2 shows the RVs of the primary and secondary determined using this algorithm at those epochs where the $\mathrm{CCF}$ is double peaked. We add $0.05 \mathrm{~km} \mathrm{~s}^{-1}$ in quadrature to the TODCOR formal errors to account for additional noise effects like wavelength calibration, small tracking induced PSF changes, etc. While the HET observed the target on 18 epochs, the secondary RVs are only reliably measured for 11 epochs. These are the epochs where the primary and secondary peaks are sufficiently separated to determine an independent RV for each. While RVs can be determined for the other seven epochs, they are RVs of blended spectra, and the associated systematic error is not only larger, but also more difficult to quantify.

Since both peaks are unambiguously detected in TODCOR at these epochs, we are also able to measure the secondary to primary flux ratio, $\alpha$, which we determine to be $\alpha=$ $0.335 \pm 0.035$ by averaging the flux ratio of the templates (G5V and K0V) over four bandpasses spanning 4663-5863 A. Finally, the mass ratio derived from these 11 epochs is $q \sim 0.88$.

\subsection{FastCam Lucky Imaging}

The MARVELS team obtained lucky imaging for TYC 3010 in order to detect any spatially resolvable companions. In 2011 April, using the FastCam (Oscoz et al. 2008) instrument on the $1.5 \mathrm{~m}$ TCS telescope at Observatorio del Teide in Spain, we obtained 47,000 frames in the I-band with a $70 \mathrm{~ms}$ exposure time for each frame. Data processing was accomplished with a custom-made IDL pipeline.

As described in Fleming et al. (2012), the best frames are selected via the brightest pixel (BP) method. The frames with the brightest $X \%$ of BPs are combined to generate a final image, where $X=\{1,5,15,30,50,80\}$ for TYC 3010 . Figure 4 shows the resulting final images for each particular percentage of the best frames.

No companions are detected, but we can place constraints on the upper limit of the masses of resolvable companions. Using the spectroscopic $T_{\text {eff }}$ for TYC 3010 (see Section 3.2.2), and the relations from Mamajek (2011), we determine the bolometric magnitude. Combining the bolometric magnitude with mass-luminosity relations (Henry et al. 1999; Henry 2004; Delfosse et al. 2000; Xia et al. 2008; Xia \& Fu 2010), we convert the detection limit for the $I$-band magnitude into a lower limit for the masses of detectable companions at different separations. At the $5 \sigma$ level, where $\sigma$ is defined in Femenía et al. (2011) as the rms of the counts within concentric annuli centered on
TYC 3010, and using 8 pixel boxes, we can rule out the presence of detectable companions above a mass of $\sim 0.35 M_{\odot}$ outside of 50 AU (see Figure 5).

\subsection{Keck AO Imaging}

In addition to the lucky imaging, we were also able to obtain adaptive optics (AO) images of TYC 3010 on 2012 October 21 UT using the NIRC2 imager at Keck (instrument PI: Keith Matthews; Matthews \& Soifer 1994). Observations consist of a sequence of nine dithered frames in the $K^{\prime}$ filter (central $\lambda=2.12 \mu \mathrm{m}$ ) using the narrow camera (plate scale $=10$ mas $\mathrm{pix}^{-1}$ ) setting. Each frame consisted of 20 coadds with $0.1814 \mathrm{~s}$ of integration time per coadd, totaling $32.65 \mathrm{~s}$ of on-source exposure time. Images were processed using standard techniques to remove hot pixels, subtract the sky-background, and align and coadd the cleaned frames. No candidate companions were identified in either raw or processed images. Figure 5 shows our sensitivity to off-axis sources as a function of angular separation. Our diffractionlimited observations rule out the presence of companions 6.5 magnitudes fainter than the primary star for separations beyond $0.5^{\prime \prime}(5 \sigma)$. Using theoretical isochrones from (Girardi et al. 2002), we convert this magnitude limit to a mass upper limit, as shown in Figure 5; we can exclude companions with a mass above $0.13 M_{\odot}$ outside of $100 \mathrm{AU}$.

\section{RESULTS}

In this section we present the orbit solution of the TYC 3010 system. First we show how the data initially suggested a spurious solution in which TYC 3010 is a single star with a BD companion. Next we present the correct solution, in which TYC 3010 is shown to be a double-lined spectroscopic stellar binary (SB2) with two solar-type stars, and we provide a full characterization of the system properties.

\subsection{Initial Spurious Solution: A BD Companion to a Solar-type Star}

Of the $28 \mathrm{RV}$ measurements collected with the MARVELS instrument, 24 passed the data quality checks and were therefore included in the automated orbit solution fitting procedures. For the ARCES data, the first 14 consecutive RV points obtained during the initial set of observations were fully consistent with our working solution, that TYC 3010 was a candidate BD (see Figure 1 and Table 4). These RV points are well fit by a solution consistent with a substellar object $\left(M \sin i \sim 50 M_{\text {Jup }}\right.$ ) orbiting in the BD desert around a solar-type star. A robust fit to the low amplitude $\left(\sim 1-2 \mathrm{~km} \mathrm{~s}^{-1}\right)$ variations was found with the EXOFAST program (Eastman et al. 2013), which uses a set of Markov Chain Monte Carlo (MCMC) trials to find the best fit. This solution, shown in Figure 1 (top panel), is a very convincing fit to the 38 originally included MARVELS (red points) and APO (blue points) measurements. This fit yielded a $\chi^{2}$ of 34.63 after scaling the error bars to force $\chi^{2} /$ dof $\sim 1$. These scalings were not unreasonable compared to other MARVELS candidates.

As noted previously, four of the original MARVELS RV measurements were initially rejected as outliers. The outlier rejection procedure included a $40 \sigma$ statistical clipping to avoid phase wrapping, and rejection of consecutive points deviating by a large systematic offset from the bulk of the measurements. The latter rejection step was specifically implemented in an attempt to account for cases of fiber mis-pluggings, which are known to happen on occasion, in which the wrong star 


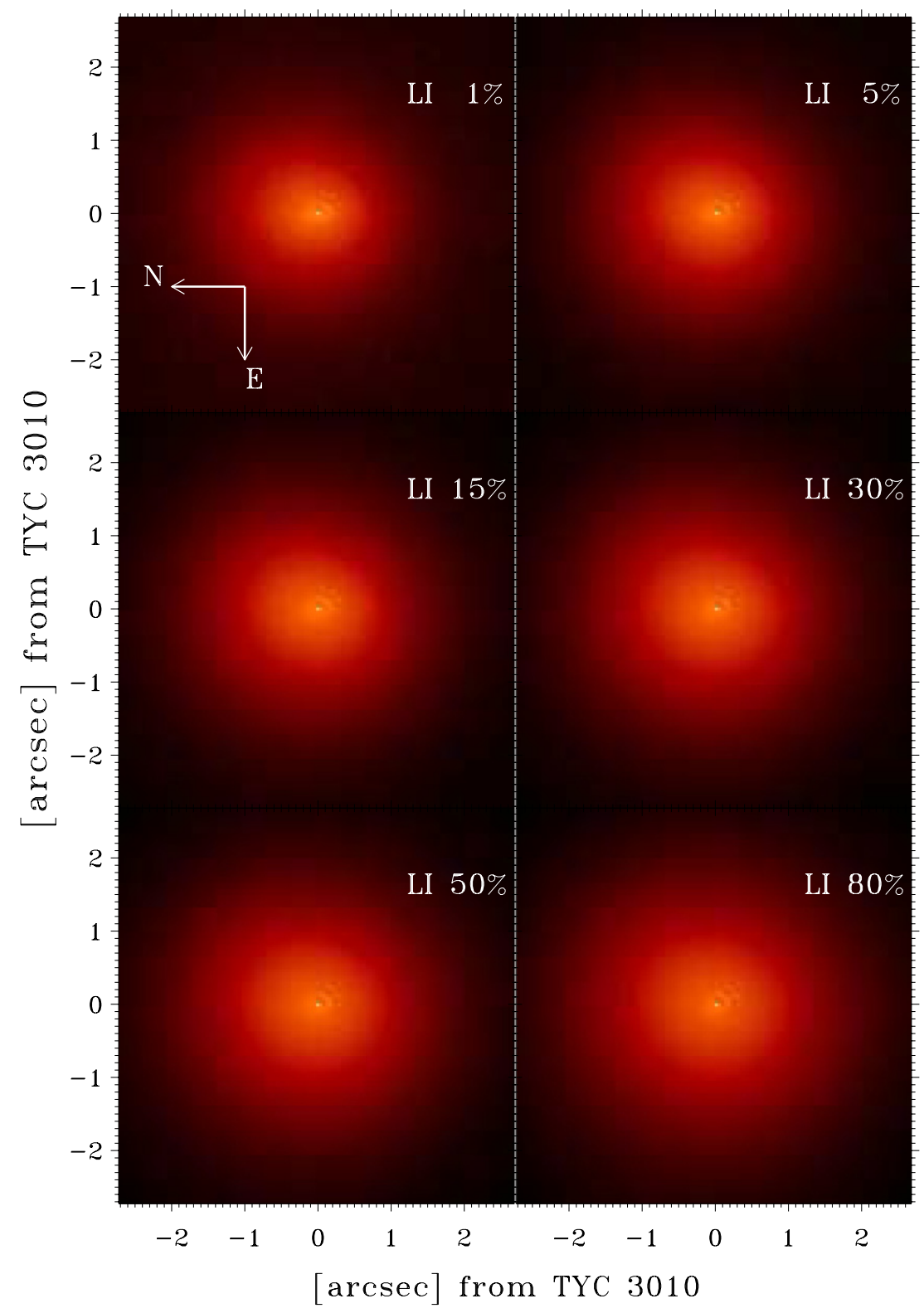

Figure 4. The best lucky imaging frames for TYC 3010. The best frames are selected according to the brightest pixel (BP) method as described in Section 2.4 . (A color version of this figure is available in the online journal.)

is observed for a few observations in a row and those few measurements appear at a very different systemic velocity relative to the majority of the measurements. The four rejected MARVELS measurements are also shown in Figure 1 (bottom panel, red points) near HJD 2455250. The final (correct) orbit solution is also shown (see details below), but it must be noted that this final orbit solution is only a good fit after properly disentangling the RVs from epochs where just a single set of spectral lines is resolved; it is not a good fit to the directly observed single-lined RV measurements, since these are in fact a flux-weighted average of the true primary and secondary RVs. The six "outlier" measurements from this first set of observations (four MARVELS points and two ARCES points) appear systematically displaced by $15-20 \mathrm{~km} \mathrm{~s}^{-1}$ relative to the other 38 measurements, which are well fit by the spurious orbit (solid curve) but not by the correct orbit (dashed curve).

In addition, as we have done with all MARVELS candidates, we performed a fit to the spectral energy distribution (SED) of the system to verify that it is consistent with a single stellar source and to provide a consistency check on the spectroscopically determined stellar properties (see below). We constructed the SED using fluxes (see Table 5) from the Tycho catalogue (Høg et al. 2000), APASS (AAVSO Photometric AllSky Survey; Data Release 6, see Henden et al. 2012), Two Micron All Sky Survey (Cutri et al. 2003), and WISE (Wright et al. 2010). NextGen models (Hauschildt et al. 1999) are used to generate theoretical SEDs by holding $T_{\text {eff }}, \log g$, and $[\mathrm{Fe} / \mathrm{H}]$ at the spectroscopically determined values (see below), and the maximum extinction $A_{\mathrm{V}}$ was limited to 0.05 mag based on the dust maps of Schlegel et al. (1998). The best fit model can be seen in the top panel of Figure 6; it corresponds to an $A_{\mathrm{V}}$ of $0.035 \pm 0.015$, and a distance of $162 \pm 35 \mathrm{pc}$. This single-star SED fit to the available photometry spanning $0.2-12 \mu \mathrm{m}$ is quite good, with the only hint of a discrepancy being a mild excess that appears in the Galaxy Evolution Explorer (GALEX) nearUV (NUV) passband, despite the lack of any strong emission 


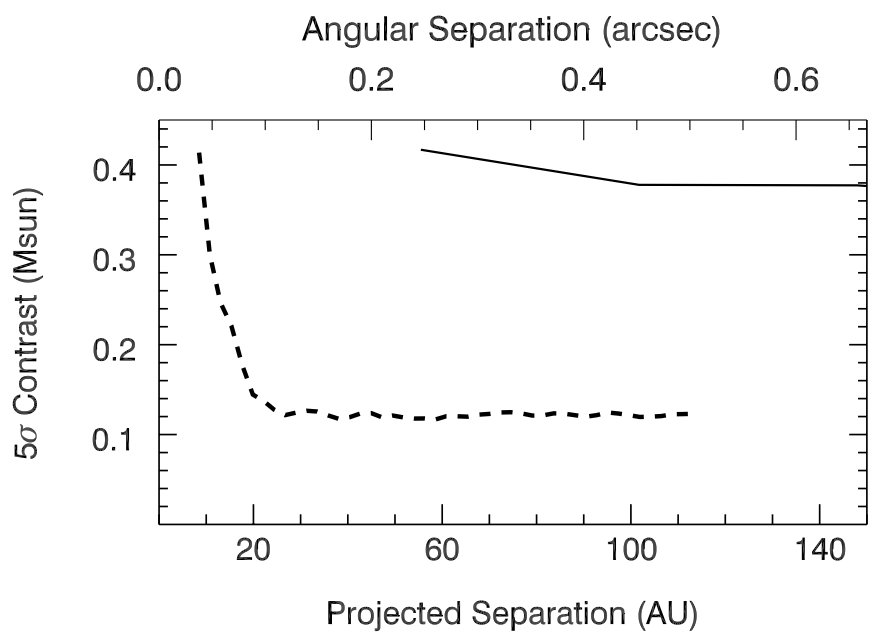

Figure 5. Detectability (contrast curve) for the lucky imaging (solid) and Keck AO (dashed) images obtained for TYC 3010. Given the lucky imaging and AO

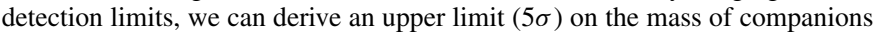
as a function of angular separation. With this upper limit, we can rule out the presence of companions above a mass of $\sim 0.35 M_{\odot}$ outside of $\sim 50 \mathrm{AU}$, and above a mass of $\sim 0.13 M_{\odot}$ outside of $\sim 100 \mathrm{AU}$.

Table 4

TYC 3010 Orbital Parameters: Spurious and True RV Solutions

\begin{tabular}{lcc}
\hline \hline & Spurious Solution & True Solution \\
\hline$T_{P}\left(\mathrm{BJD}_{\mathrm{TDB}}-2450000\right)$ & $5496.8_{-2.0}^{+1.8}$ & $5970.04 \pm 5.1$ \\
$P$ (days) & $238.49_{-0.70}^{+0.73}$ & $237.96 \pm 0.04$ \\
$e$ & $0.384_{-0.048}^{+0.067}$ & $0.785 \pm 0.003$ \\
$\omega(\mathrm{deg})$ & $200.88_{-2.58}^{+2.35}$ & $188.86 \pm 0.67$ \\
$K_{1}\left(\mathrm{~km} \mathrm{~s}^{-1}\right)$ & $1.970_{-0.130}^{+0.240}$ & $15.38 \pm 0.25$ \\
$K_{2}\left(\mathrm{~km} \mathrm{~s}^{-1}\right)$ & $\ldots$ & $17.50 \pm 0.16$ \\
$\gamma\left(\mathrm{km} \mathrm{s}^{-1}\right)$ & $61.759_{-0.087}^{+0.077}$ & $61.28 \pm 0.09$ \\
$q=M_{\mathrm{B}} / M_{\mathrm{A}}$ & $\ldots$ & $0.88 \pm 0.02$ \\
\hline
\end{tabular}

Notes. The spurious solution consists of the EXOFAST (Eastman et al. 2013) fit to the MARVELS and ARCES RV data, excluding the points initially thought to be invalid outliers. The true solution was determined with the BINARY software (Gudehus 2001) and the MARVELS, ARCES, and HRS observations. For the true (SB2) solution, the single-lined RV measurements were disentangled into their primary and secondary components (see Section 3.2.1).

Table 5

Catalog Properties of TYC 3010-1494-1

\begin{tabular}{lccl}
\hline \hline Parameter & Value & Uncertainty & \multicolumn{1}{c}{ Reference } \\
\hline$\alpha(2000)$ & 110011.45 & & Høg et al. (2000) \\
$\delta(2000)$ & +394324.74 & & Høg et al. (2000) \\
pmRA $\left(\right.$ mas yr $\left.^{-1}\right)$ & -43.4 & 1.7 & Høg et al. (2000) \\
pmDE $\left(\operatorname{mas~yr}^{-1}\right)$ & 3.3 & 1.6 & Høg et al. (2000) \\
$B_{\mathrm{T}}$ & 13.102 & 0.297 & Høg et al. (2000) \\
$V_{\mathrm{T}}$ & 11.758 & 0.143 & Høg et al. (2000) \\
$B$ & 12.007 & 0.153 & Henden et al. (2012) \\
$V$ & 11.367 & 0.145 & Henden et al. (2012) \\
$I_{\mathrm{C}}$ & 10.531 & 0.074 & Henden et al. (2012) \\
$g$ & 11.579 & 0.177 & Henden et al. (2012) \\
$r$ & 11.093 & 0.089 & Henden et al. (2012) \\
$i$ & 10.870 & 0.127 & Henden et al. (2012) \\
$J$ & 9.977 & 0.021 & Cutri et al. (2003) \\
$H$ & 9.554 & 0.016 & Cutri et al. (2003) \\
$K_{s}$ & 9.488 & 0.019 & Cutri et al. (2003) \\
WISE1 $(3.4 \mu \mathrm{m})$ & 9.407 & 0.006 & Wright et al. (2010) \\
WISE2 $(4.6 \mu \mathrm{m})$ & 9.482 & 0.006 & Wright et al. (2010) \\
WISE3 $(12 \mu \mathrm{m})$ & 9.470 & 0.038 & Wright et al. (2010) \\
\hline
\end{tabular}
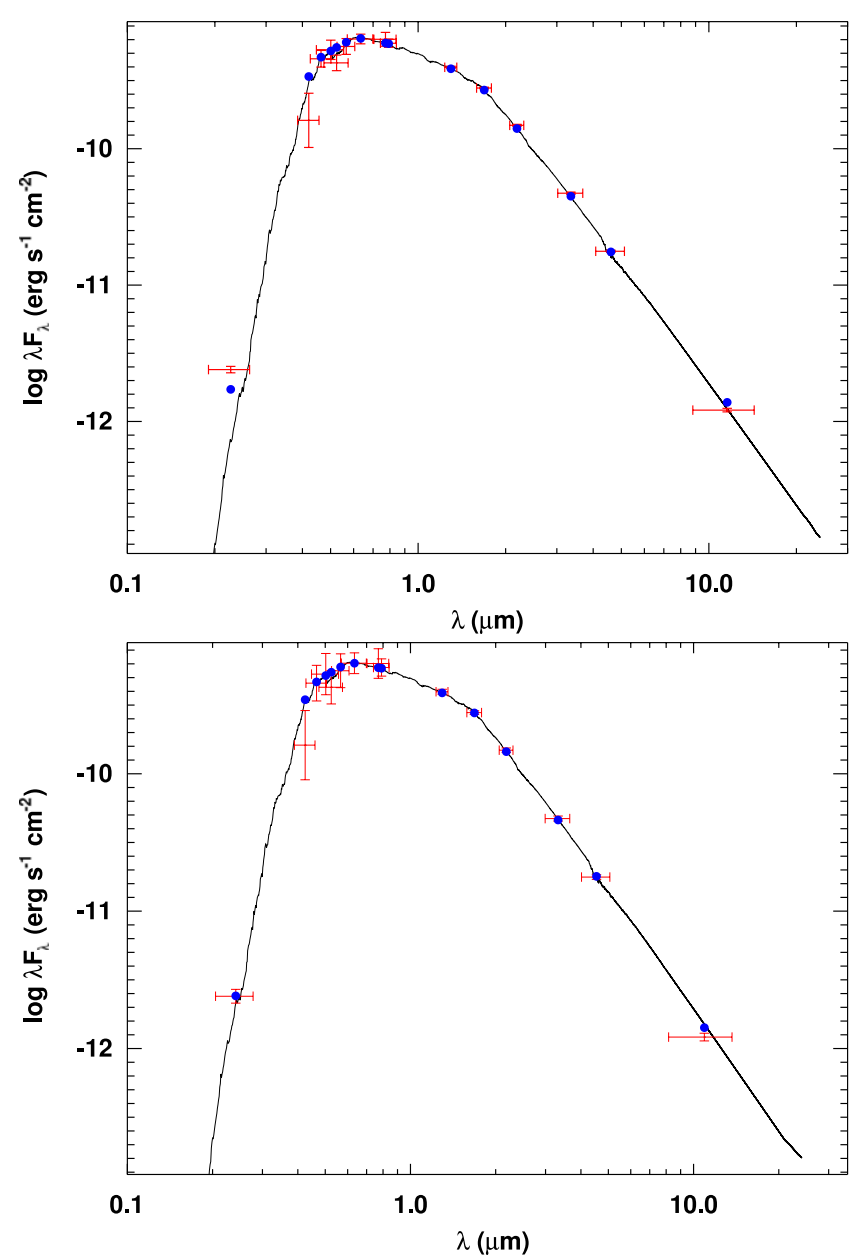

Figure 6. Top: A NextGen model atmosphere (solid line) fit to the observed broadband fluxes for TYC 3010 (assuming a single stellar component). The blue points are the flux values predicted by the model for the different bandpasses. The vertical red bars correspond to the uncertainties in the measured fluxes, while the horizontal red bars are the approximate widths of the bandpasses. This fit assumed that TYC 3010 was a single star, and found that $T_{\text {eff }}=5400 \pm 100 \mathrm{~K}$, $\log g=4.5 \pm 0.5,[\mathrm{Fe} / \mathrm{H}]=0.0 \pm 0.1$, and $A_{V}=0.035 \pm 0.015$, yielding a distance of $162 \pm 35$ pc. Bottom: A second NextGen fit that uses two stellar components (corresponding to the primary and secondary stars of TYC 3010) with one of the components constrained to the spectroscopically determined stellar parameters for the primary $\left(T_{\text {eff }}=5589 \pm 148 \mathrm{~K}, \log g=4.68 \pm 0.44\right.$, $[\mathrm{Fe} / \mathrm{H}]=0.09 \pm 0.20$ ). This fit estimates the secondary stellar parameters to be $T_{\text {eff }}=4600 \pm 850 \mathrm{~K}, R=0.75 \pm 0.4 R_{\odot}, \log g=4.6 \pm 0.2$, and the distance to TYC 3010 to be $225 \pm 40 \mathrm{pc}$, with an $A_{V}=0.03 \pm 0.02\left(\chi^{2} /\right.$ dof $\left.=0.75\right)$.

(A color version of this figure is available in the online journal.)

in the observed $\mathrm{Ca} \mathrm{HK}$ lines. However, this by itself was not deemed to be a compelling reason to suspect the high quality orbit solution.

Thus, at this point in our analysis, fully $38 \mathrm{RV}$ measurements from two separate instruments were well fit by the same orbit solution of a single, solar-type star with a $\sim 50 M_{\text {Jup }}$ companion on a modestly eccentric orbit. The SED of TYC 3010 was furthermore consistent with being a single solar-type star, and the lack of any companions in the high-resolution imaging ruled out a blend scenario in which the RV variations might be caused by a binary beyond 0'.5 of the line of sight. Only four of the discovery RV measurements appeared to be discrepant, and these were rejected for what appeared to be good reasons, behaving not unlike fiber mis-pluggings that the MARVELS team had observed in other stars before. However, the last two RV measurements from the first set of ARCES 


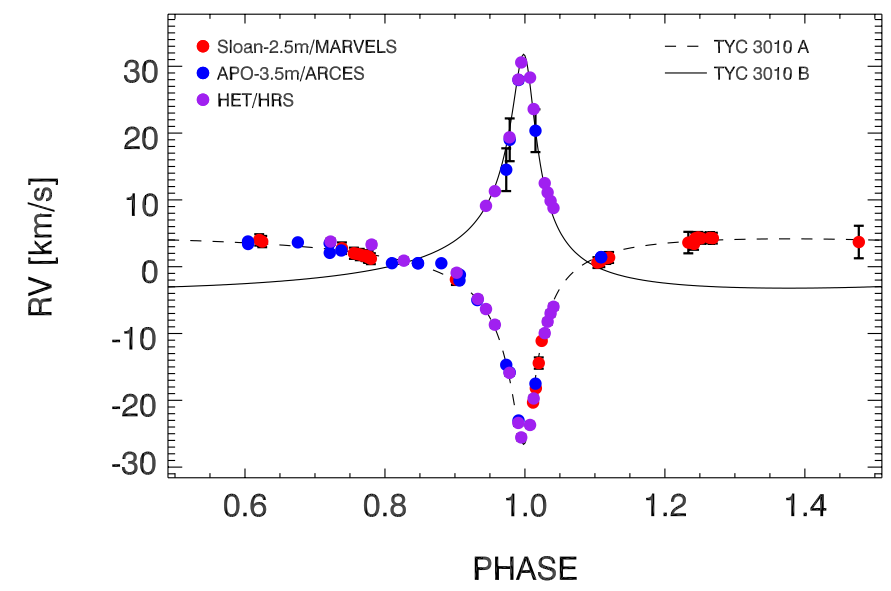

Figure 7. The correct phase-folded radial velocity curve for TYC 3010. The best-fit BINARY (Gudehus 2001) orbital solution for the primary (dashed line) and secondary (solid line) are shown with the RVs obtained from the MARVELS (red), ARCES (blue), and HRS (purple) spectrographs. This solution corresponds to a period of $\sim 238$ days, an eccentricity of $\sim 0.79$, with $K_{1} \sim 15.38 \mathrm{~km} \mathrm{~s}^{-1}$ and $K_{2} \sim 17.50 \mathrm{~km} \mathrm{~s}^{-1}$. Finally, for the RV points outside of periastron, it was necessary to de-blend the observed RVs with the method described in Section 3.2.1.

(A color version of this figure is available in the online journal.)

observations appeared as strong outliers (see Figure 1, blue points near HJD 2455730). As they were observed with a standard echelle spectrograph, these could not be attributed to fiber mis-pluggings, and inspection of the CCFs revealed double lines (see bottom panel of Figure 3), immediately nullifying the $\mathrm{BD}$ companion hypothesis.

\subsection{Final Solution: A Highly Eccentric, Double-lined Spectroscopic Binary}

To further confirm that TYC 3010 was indeed a stellar binary, we closely observed the next peripassage with the HRS spectrograph on HET. With HET, we obtained complete coverage of periastron, permitting a complete double-lined orbit solution. In this section we present the correct orbit solution for TYC 3010, including all the points from the discovery and subsequent data, which shows that TYC 3010 is an SB2 with a period of $P \sim 238$ days, an eccentricity of $e \sim 0.79$, and a mass ratio of $q \sim 0.88$. With this eccentricity and orbital period, TYC 3010 lies near the upper bound of (but within) the distribution of orbital eccentricities of solar-type binaries with orbital periods of 100-300 days (see, e.g., Duquennoy \& Mayor 1991; Raghavan et al. 2010). The orbital parameters for the binary are summarized in Table 4, the RV solution is shown in Figure 7, and a schematic of the orbit is shown in Figure 8. In this section we also describe our determination of the stellar parameters for the primary in TYC 3010, and we estimate its mass and radius using the relations described in Torres et al. (2010). Since the secondary is comparable in mass to the primary, we had to take special care in accounting for the flux contamination from the secondary, both in our determination of the stellar parameters and with the RV values that we measured for the system outside of periastron.

\subsubsection{RV Fitting}

For the orbital solution of the binary, we used the RV fitting software described in Gudehus (2001). Since we do not resolve two sets of spectral lines for the phases outside of periastron, most of the RV points correspond to a flux-weighted average of the primary and secondary RVs. In order to de-blend the fluxweighted RVs that we measured, and derive the corresponding primary RVs, we used the following prescription.

We treat the blended velocities as a flux-weighted average of the primary and secondary velocities:

$$
v_{\text {blend }}=\frac{v_{A} F_{A}+v_{B} F_{B}}{F_{A}+F_{B}},
$$

where $v_{A}$ and $v_{B}$ are the primary and secondary velocities respectively, and $F_{A}$ and $F_{B}$ are the primary and secondary fluxes. We normalize the flux weights by setting the sum of the fluxes, $F_{A}+F_{B}$, to unity. Using the flux ratio, $\alpha=F_{B} / F_{A}$, from the TODCOR analysis (which was only performed for the HET/HRS epochs where it was possible to resolve two sets of spectral lines), we can solve for $F_{A}$ and $F_{B}$ in terms of $\alpha$ :

$$
F_{A}=\frac{1}{1+\alpha} ; \quad F_{B}=\alpha F_{A} .
$$

In addition, we can use the mass ratio, $q=M_{B} / M_{A}$, from the $\mathrm{RV}$ solution to write $v_{B}$ in terms of $v_{A}$, since $M_{B} / M_{A}=v_{A} / v_{B}$.

$$
v_{B}=-v_{A}\left(\frac{M_{A}}{M_{B}}\right)=\frac{-v_{A}}{q}
$$

Returning to (1), we can now write

$$
v_{A}=\frac{v_{\text {blend }}}{F_{A}-F_{B} / q}=\left(\frac{1+\alpha}{1-\alpha / q}\right) v_{\text {blend }} .
$$

With Equation (4), we can iteratively solve for a final set of deblended RVs for the primary. For the first iteration, we provide an initial guess for $q$ by performing a joint fit to the primary RVs (blended+unblended) combined with the secondary RVs (unblended; only measured during periastron). Inserting this initial guess for $q$ into Equation (4), we derive an initial set of de-blended primary RVs. Then we perform another joint fit to the primary (de-blended+unblended) and secondary (unblended) RVs to refine our value for $q$. We repeat the process until $q$ converges. The value we find for $q(0.878 \pm 0.016)$ from this de-blending analysis is in excellent agreement with the value for $q(\sim 0.88)$ that we found from the ratio of the primary and secondary RVs that were measured for the $11 \mathrm{HET} / \mathrm{HRS}$ epochs where two peaks were resolved in the CCFs. Thus, $q$ has been determined very precisely by the orbital solution (better than $3 \%$ ), and is more precise than the individual quoted errors on the masses.

As a further consistency check on $\alpha$ and $q$, we also note that according to the relationship between mass and bolometric luminosity from Torres et al. (2010), there should be a relationship between $\alpha$ and $q$. Since $\alpha$ is derived from a set of finite wavelength bands, it is not bolometric. However, since the stars have temperatures that are not too dissimilar, $\alpha$ is likely to be approximately equal to the ratio of the bolometric luminosities. For stars with $M=0.6-1.2 M_{\odot}$, a fit to the Torres et al. (2010) data yields $L \propto M^{5.1}$. Thus, $\alpha=q^{5.1}$, so $q \sim(0.335)^{1 / 5.1} \sim 0.81$, which is within $3 \sigma$ of the value obtained from the RV analysis.

\subsubsection{Determining the Stellar Parameters for TYC 3010}

The stellar parameters for the primary were determined with a double-lined spectrum obtained near periastron (see Section 2.2). The spectroscopic analysis used to determine the atmospheric parameters is similar to the one described 


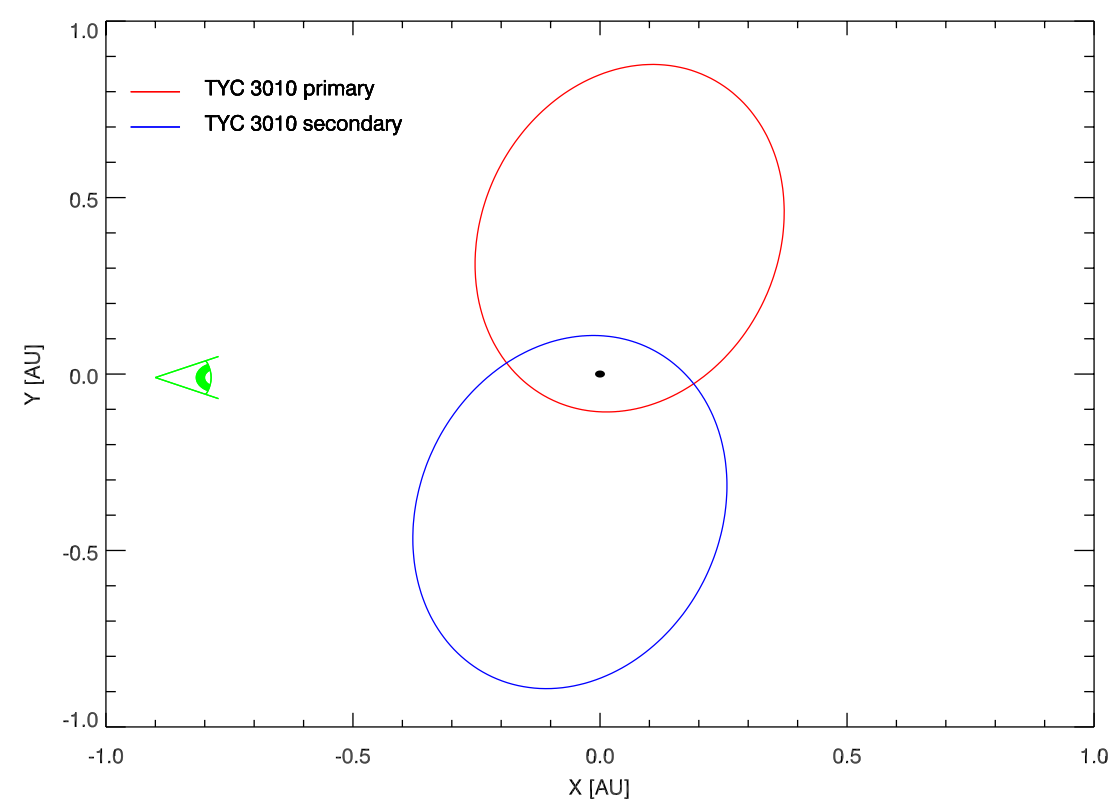

Figure 8. A schematic of the TYC 3010 system drawn to scale, showing the primary (red) and secondary (blue) orbits in the orbital plane. The position of the center of mass of the system is marked by the black point. Given the eccentricity $(e \sim 0.79)$ and the fact that the semi-major axis is aligned nearly perpendicular to the line of sight $\left(\omega \sim 189^{\circ}\right)$, for a substantial fraction of the orbit the system can mimic the RV signal that would normally be induced by a secondary object with a minimum mass in the brown dwarf regime. Coupled with the relatively long period ( 238 days), depending on the frequency of the observations, it can be fairly easy to miss peripassage during a given orbit.

(A color version of this figure is available in the online journal.)

in Wisniewski et al. (2012), where we use two independent methods that require the conditions of excitation and ionization equilibria for $\mathrm{Fe}$ I and $\mathrm{Fe}$ II lines. These methods are referred to as the "BPG" (Brazilian Participation Group) method and the "IAC" (Instituto de Astrofísica de Canarias) method.

The "BPG" analysis was done in local thermodynamic equilibrium (LTE) using the 2002 version of MOOG $^{19}$ (Sneden 1973) and one-dimensional plane-parallel model atmospheres interpolated from the ODFNEW grid of ATLAS9 models (Kurucz 1993; Castelli \& Kurucz 2004). In previous MARVELS papers (e.g., Wisniewski et al. 2012 and references therein), the equivalent widths (EWs) of the $\mathrm{Fe}$ lines were determined in an automated fashion. However, in this case, the EWs were manually measured to carefully account for visible blends on the Fe lines from the secondary's spectrum. We note that contaminations from very weak lines could have affected the EW measurements. In order to correct the EWs measured for the primary for the veiling from the continuum flux of the secondary star, we followed a procedure similar to the one described in Section 5.2.1 of González Hernández et al. (2008). According to their prescription, we can relate the value of the true equivalent width $\left(\mathrm{EW}_{\text {true }}\right)$ of a given line to the observed equivalent width $\left(\mathrm{EW}_{\mathrm{obs}}\right)$ through the following relationship,

$$
\mathrm{EW}_{\text {true }, \mathrm{A}}=f_{\mathrm{A}}\left(\mathrm{EW}_{\mathrm{obs}, \mathrm{A}}\right),
$$

where $f_{\mathrm{A}}$ is the so-called veiling factor for the primary. The veiling factors for the two components are related by

$$
\frac{f_{\mathrm{B}}(\lambda)}{f_{\mathrm{A}}(\lambda)}=\frac{F_{\mathrm{A}}(\lambda)}{F_{\mathrm{B}}(\lambda)}=\frac{1}{\alpha}
$$

\footnotetext{
19 http://www.as.utexas.edu/ chris/moog.html
}

Table 6

TYC 3010 Properties Derived by This Work

\begin{tabular}{lcc}
\hline \hline & System Properties & \\
\hline Parameter & Value & Uncertainty \\
$\alpha=F_{\mathrm{B}} / F_{\mathrm{A}}$ & 0.335 & 0.035 \\
$q=M_{\mathrm{B}} / M_{\mathrm{A}}$ & 0.878 & 0.016 \\
$A_{V}$ & 0.03 & 0.02 \\
$d(\mathrm{pc})$ & 225 & 40 \\
\hline & TYC $3010 \mathrm{~A}$ & TYC $3010 \mathrm{~B}$ \\
\hline$T_{\text {eff }}(\mathrm{K})$ & $5589 \pm 148$ & $4600 \pm 850$ \\
$\log g(\mathrm{cgs})$ & $4.68 \pm 0.44$ & $4.60 \pm 0.20$ \\
{$[\mathrm{Fe} / \mathrm{H}]$} & $0.09 \pm 0.20$ & $\ldots$ \\
$M\left(M_{\odot}\right)$ & $1.01_{-0.11}^{+0.14}$ & $0.73_{-0.23}^{+0.24}$ \\
$R\left(R_{\odot}\right)$ & $0.77_{-0.27}^{+0.53}$ & $0.68_{-0.18}^{+0.23}$ \\
\hline
\end{tabular}

Notes. The properties for the primary were determined by the spectroscopic stellar parameters and the Torres et al. (2010) relations. The properties for the secondary were determined from the stellar parameters found by the twocomponent fit to the SED and the Torres relations.

where $F_{\mathrm{A}}$ and $F_{\mathrm{B}}$ are the fluxes for the primary and secondary. Furthermore, the veiling factors satisfy the equation

$$
\frac{1}{f_{\mathrm{A}}(\lambda)}+\frac{1}{f_{\mathrm{B}}(\lambda)}=1
$$

To simplify our analysis, we treated the veiling factors and flux ratio as if they were wavelength independent. Using the average flux ratio derived by TODCOR $\left(\alpha=F_{B} / F_{A}=0.335 \pm 0.035\right.$; see Section 2.3.2), and the added constraint from Equation (7), we find the veiling factor for the primary to be $f_{\mathrm{A}} \sim 1.34$. Thus, after correcting the EWs, we find the stellar parameters to be $T_{\text {eff }}=5589 \pm 148 \mathrm{~K}, \log g=4.68 \pm 0.44$, and $[\mathrm{Fe} / \mathrm{H}]=$ $0.09 \pm 0.20$ (see Table 6 ). The uncertainties for these parameters are larger than the typical errors that we achieve with our 
spectroscopic analysis because of the flux contamination from the secondary star.

The "IAC" analysis extracted the stellar parameters of the primary and secondary stars by considering veiling factors that were wavelength-dependent. These veiling factors are estimated using low-resolution Kurucz fluxes (Allende Prieto \& Lambert 2000 and references therein) and the following equation:

$$
\frac{f_{\mathrm{B}}(\lambda)}{f_{\mathrm{A}}(\lambda)}=\frac{\Gamma_{\mathrm{A}}(\lambda)}{\Gamma_{\mathrm{B}}(\lambda)}\left(\frac{R_{\mathrm{A}}}{R_{\mathrm{B}}}\right)^{2},
$$

where $\Gamma_{\mathrm{A}}$ and $\Gamma_{\mathrm{B}}$ correspond to the surface brightness of the primary and the secondary respectively. To determine the ratio of the radii, we derived an empirical mass-radius relationship from a sample of 55 stars from Torres et al. (2010), with the masses restricted to $0.7 M_{\odot}<M<1.4 M_{\odot}$. We fit a function to the data of the form

$$
\log R / R_{\odot}=a \log \left(M / M_{\odot}\right)+b,
$$

where $a=1.052 \pm 0.097$ and $b=0.036 \pm 0.008$. Thus, the ratio of the radii for the components of TYC 3010 can be written as

$$
R_{\mathrm{A}} / R_{\mathrm{B}}=\left(M_{\mathrm{A}} / M_{\mathrm{B}}\right)^{1.052} \text {. }
$$

The mass ratio was determined from the TODCOR analysis to be $q=M_{B} / M_{A} \sim 0.88$, so we find that $R_{A} / R_{B}=1.142$.

As a first guess, we adopt the above values to estimate the stellar mass and radius of the primary (Allende Prieto et al. 2004; Reddy et al. 2006; Ramírez et al. 2007), from solarscaled theoretical isochrones (Bertelli et al. 1994). The mass ratio allows us to derive a first guess of the $T_{\text {eff, } \mathrm{B}}$ value for the secondary to be roughly $5100 \mathrm{~K}$, assuming $\log g \sim 4.70$ and the same metallicity as the primary. The stellar radii we get from the comparison with isochrones are $0.89 R_{\odot}$ and $0.77 R_{\odot}$, and thus the ratio is $R_{A} / R_{B}=1.145$, which is very similar to the value previously estimated $\left(R_{A} / R_{B}=1.142\right)$. Thus, the derived veiling factors lie in the range $f_{\lambda, A} \sim 1.45-1.55$ and $f_{\lambda, B} \sim 3.20-2.85$ in the spectral region $4500-7000 \AA$.

We then measure automatically, using the code ARES (Sousa et al. 2007), the EWs of the Fe I and Fe II lines (Sousa et al. 2008) for both stellar components and correct them using the wavelength-dependent veiling factors. We then use the code STEPAR (Tabernero et al. 2012) to automatically derive the stellar parameters of each component and we get $T_{\text {eff, } A}=$ $5410 \pm 124 \mathrm{~K}, \log g_{A}=4.57 \pm 0.56,[\mathrm{Fe} / \mathrm{H}]_{A}=0.02 \pm 0.20$ and $\xi_{A}=0.90 \pm 0.22$ from $162 \mathrm{Fe}$ I and $18 \mathrm{Fe}$ II lines. The uncertainties are unexpectedly large and may be due to the contamination of neighboring lines of other elements of the companion star. Thus the results for the secondary are fairly tentative and the errors are even larger. We were only able to measure $64 \mathrm{Fe}$ I and $3 \mathrm{Fe}$ II lines to get $T_{\text {eff, } B}=5136 \pm 323 \mathrm{~K}$, $\log g_{B}=4.71 \pm 0.88,[\mathrm{Fe} / \mathrm{H}]_{B}=-0.15 \pm 0.26$ and $\xi_{B}=$ $0.75 \pm 0.40$. Compared to the "BPG" analysis, the lower $T_{\text {eff, } A}$ of the primary may be related to the different methods used to derive the veiling factors. Nevertheless, the "IAC" stellar parameters for the primary star are very similar to those previously derived and are actually consistent within the large uncertainties so we decide to adopt the "BPG" values.

With the "BPG" stellar parameters for the TYC 3010 primary, we again performed a fit to the observed SED of the system as in Section 3.1, but now also including the contribution of the secondary star. Once again, NextGen models (Hauschildt et al. 1999) are used to generate theoretical SEDs by holding $T_{\text {eff }}$,

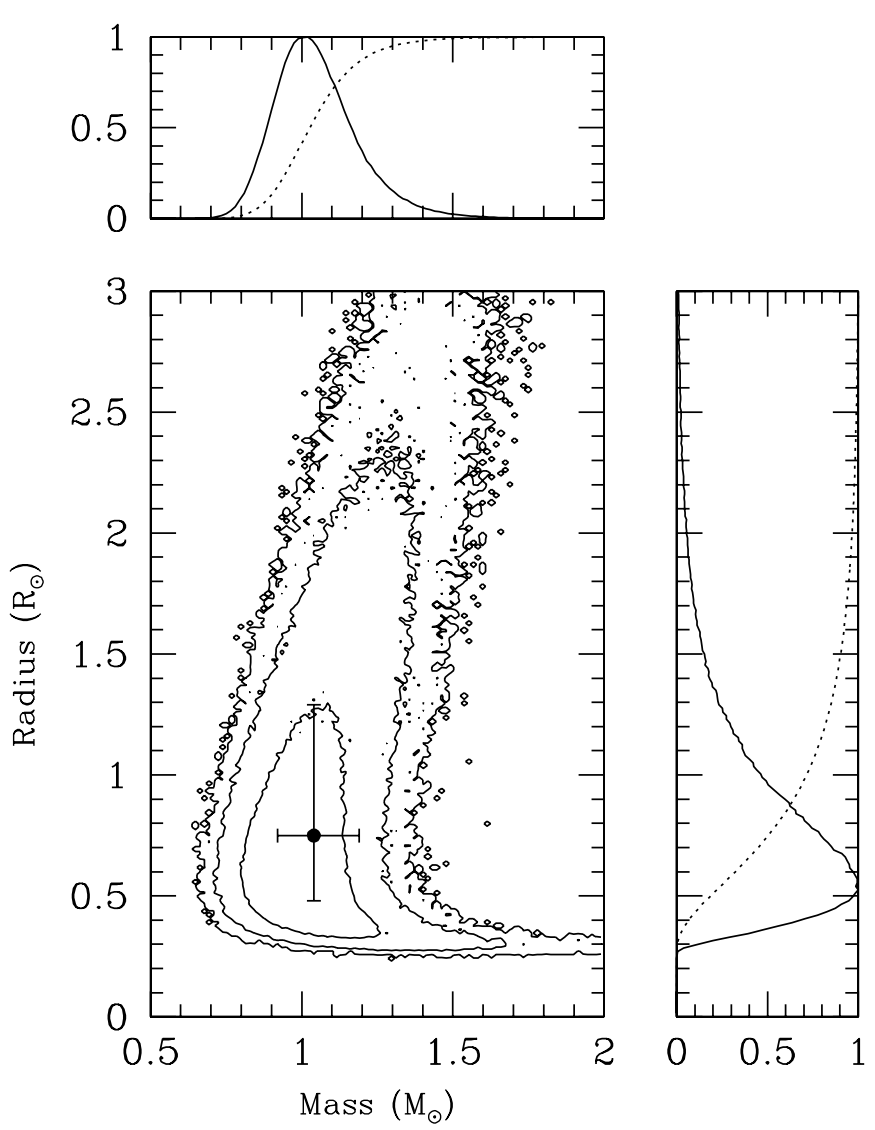

Figure 9. Mass and radius distributions for the primary component of TYC 3010. These distributions were determined by a set of MCMC trials with the spectroscopic stellar parameters and the empirical relations from Torres et al. (2010). The black point represents the median $\left(M_{\star}=1.04_{-0.12}^{+0.15} M_{\odot}, R_{\star}=\right.$ $\left.0.75_{-0.27}^{+0.54} R_{\odot}\right)$, and the error bars correspond to the $68.27 \%$ confidence intervals The contours are lines of equal probability density which enclose $68 \%, 90 \%$, and $95 \%$ of the cumulative probability relative to the maximum of the probability density. In the top and right panels, the probability distribution (solid line) and cumulative probability (dashed line) are shown for the mass and radius respectively.

$\log g$, and $[\mathrm{Fe} / \mathrm{H}]$ at the spectroscopically determined values for the primary, while the $T_{\text {eff }}$ for the secondary is found by the value that minimizes $\chi^{2}\left(\chi^{2} /\right.$ dof $\left.=0.75\right)$. The best fit model can be seen in the bottom panel of Figure 6 ; it corresponds to an $A_{\mathrm{V}}$ of $0.03 \pm 0.02$, and a distance of $225 \pm 40 \mathrm{pc}$. Compared to the SED fit performed in Section 3.1, which assumed a single stellar contribution, this two-component SED fit no longer exhibits an excess in the GALEX NUV passband, and more generally is an excellent fit to all of the available photometry. Finally, from this two-component fit to the SED, we also obtain a set of values for the stellar parameters of the secondary of TYC 3010. We find that $T_{\text {eff }}=4600 \pm 850 \mathrm{~K}, \log g=4.6 \pm 0.2$, and $[\mathrm{Fe} / \mathrm{H}]=$ $0.05 \pm 0.19$.

\subsection{Inferred Evolutionary Status of TYC 3010}

Given the spectroscopic stellar parameters, we can derive the mass and radius of the TYC 3010 primary star using the empirical relationships described in Torres et al. (2010). Figure 9 shows the result of a set of MCMC trials for the best estimate of the mass and radius. For the precise parameters of the primary $\left(T_{\text {eff }}=5589 \mathrm{~K}, \log g=4.68,[\mathrm{Fe} / \mathrm{H}]=0.09\right)$, the Torres relations give $0.98 M_{\odot}$ and $0.75 R_{\odot}$. Once one includes the fairly large uncertainties in the stellar parameters, the median values 


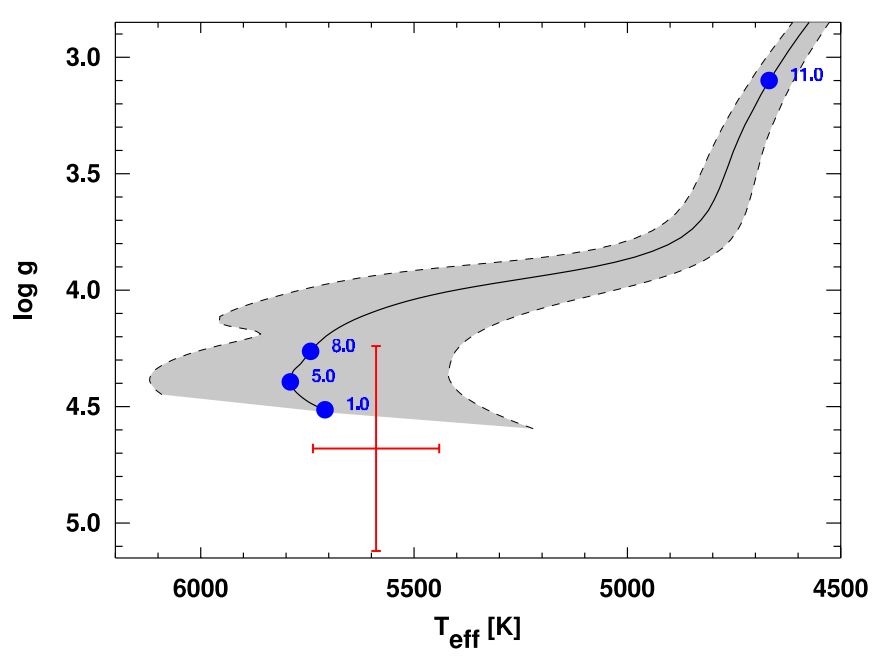

Figure 10. H-R diagram that compares the derived stellar parameters for the primary of TYC 3010 (red error bars) to a Yonsei-Yale stellar evolutionary track (solid curve; Demarque et al. 2004) for a star with a mass of $1.04 M_{\odot}$ and $[\mathrm{Fe} / \mathrm{H}]=0.09$. Ages (in Gyr) of 1.0, 5.0, 8.0, and 11.0 are represented by blue dots, and the $1 \sigma$ deviations from the evolutionary track are shown in the shaded region.

(A color version of this figure is available in the online journal.)

for the mass and radius become $1.04_{-0.12}^{+0.15} M_{\odot}$ and $0.75_{-0.27}^{+0.54} R_{\odot}$, respectively. The means are $1.05 \pm 0.15 M_{\odot}$ and $0.90 \pm 0.54 R_{\odot}$, so the distributions are quite skewed as shown in Figure 9. Compared to a Yonsei-Yale evolutionary track (see Figure 10), we do not have a strong constraint on the age, but TYC 3010 is unlikely to have evolved off the main sequence.

We can also derive the mass and radius for the secondary given the stellar parameters determined from the two-component SED fit and the Torres et al. (2010) relations. We find that $M_{B}=0.74_{-0.23}^{+0.26} M_{\odot}$ and $R_{B}=0.76_{-0.19}^{+0.27}$. This value for the mass of the secondary agrees within $1 \sigma$ of the value that can be derived using the primary mass we determined above and the mass ratio from the RV solution, i.e., $M_{B} \sim 0.89 M_{\odot}$.

\section{DISCUSSION}

\subsection{Why We Initially Derived a Spurious Solution}

The RV signal from TYC 3010 initially seemed to indicate that it was a BD orbiting a solar-type star in the BD desert. Over $80 \%$ of the MARVELS discovery data agreed with this interpretation, and there seemed to be plausible reasons for excluding the outliers. However, once similar outliers were found in the subsequent observations, we began to suspect the validity of the BD interpretation. In this section, we discuss in detail why we initially favored the BD interpretation, as well as how this conclusion was abruptly overturned by a few surprising data points.

In the discovery data, there were four outliers in total, each offset by $\sim 20 \mathrm{~km} \mathrm{~s}^{-1}$ from the rest of the data. The most anomalous of the outliers was extracted from a spectrum with a low $\mathrm{S} / \mathrm{N}$, so its $\mathrm{RV}$ value did not seem trustworthy. The remaining outliers (considering that they corresponded to a $\sim 20 \mathrm{~km} \mathrm{~s}^{-1}$ offset in RV that was only captured once during the three orbits contained in the discovery data), also seemed likely to be spurious. The MARVELS spectrograph is a fiberfed spectrograph that can observe 60 objects simultaneously. Each fiber is plugged by hand to observe the correct target, and occasionally a mistake may occur. Indeed, the MARVELS data vetting procedures were evolved to specifically include an outlier rejection step that sought to mitigate such errors, by searching for consecutive strings of measurements that were offset from the bulk of the data in a similar fashion to how these four measurements behave.

Remarkably, excluding these few apparent "outliers"-and in fact only by excluding them-permits a convincing orbit solution. It is not intuitive that this should be the case, in particular because only $\sim 15 \%$ of the measurements are excluded (including both the discovery data and the initial follow-up data which appeared to corroborate the spurious solution) and because the resulting solution is so dramatically different from the true solution. Evidently, a system such as TYC 3010 (with its extreme eccentricity, leading to punctuated large RV excursions, and its orbital orientation being nearly perpendicular to the line of sight, leading to very small RV variations for $\sim 95 \%$ of the orbit) is able to mimic a more circular orbit of a low-mass companion about a single star. Moreover, the similarity of the two stars in TYC 3010 leads to a combined light SED that is only slightly different from that of a single star at a nearer distance.

Thus many lines of evidence supported the initial solution, considering that the BD interpretation appeared to be supported by two years of discovery RV data, six months of additional RV observations, lucky imaging, and a well-constrained SED. Indeed, when the two follow-up RV measurements observed near periastron appeared, indicating a possible problem with the original orbit solution, we began to search for reasons to suspect the validity of these two anomalous points. At first, we thought the situation might be similar to the fiber mis-pluggings believed to have occurred with the discovery data, and we considered that the ARCES outliers were the result of pointing at the wrong star. But after investigating the data from those two nights, we confirmed that we had observed the correct target. Next we learned of a recent change that had been made to the ARCES instrument: the ThAr lamp had recently been replaced. The ThAr lamp is used to perform the wavelength calibration, and it was plausible that the new lamp might have caused problems with the wavelength solution. Therefore, the ARCES outliers may have merely been the result of an artificial Doppler shift generated by an incorrect wavelength solution. In the end, we were only able to accept that the BD interpretation was incorrect after we inspected the CCF for each of the outliers. The CCFs for the outliers both showed two peaks instead of one, indicating the presence of a second stellar component. Furthermore, the secondary peak was comparable in height to the primary peak (see bottom panel of Figure 3), which led us to suspect that TYC 3010 was in fact a spectroscopic stellar binary.

But how did most of the data that we had for TYC 3010 conspire to imply that it was a much less massive system? The period, shape, and orientation of the orbit with respect to the line of sight (see Figure 8) made it such that for most of the orbit the two stars possess relatively low RVs with respect to each other. In particular, the difference between the magnitude of their RVs is smaller than the typical CCF width for our instruments, resulting in their CCF peaks being blended into one. Since the flux ratio is not too different from unity, and the mass ratio is also close to unity, for epochs where the spectral lines are blended, there is a near-cancellation (or strong suppression) of the true orbital velocities for the primary and secondary, which are nearly equal in magnitude but oppositely signed (see Equation (4), and recall that $v_{\text {blend }}$ is what we actually measure). Thus, for $\sim 95 \%$ of the orbit, the amplitude of the variations $\left(\sim 1-2 \mathrm{~km} \mathrm{~s}^{-1}\right)$ suggest a BD companion to a solar-type star; furthermore, the eccentricity and the orbital period ensure that 


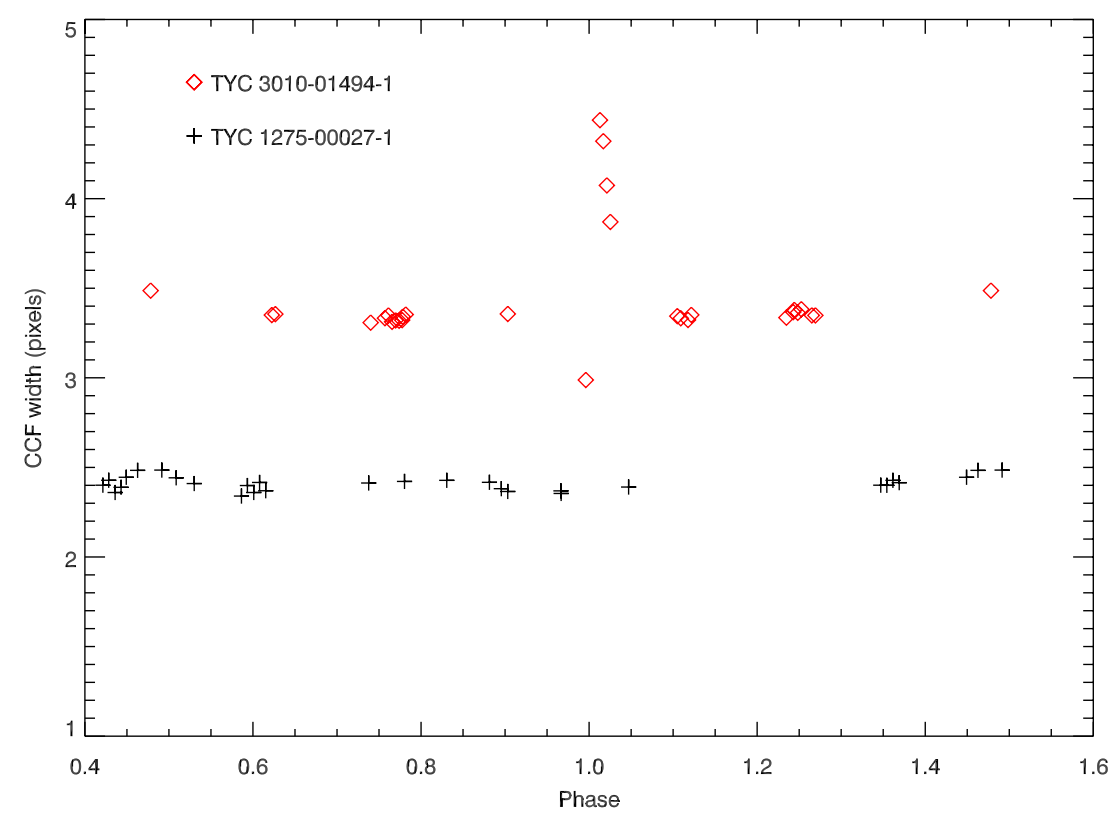

Figure 11. A comparison of how the width of the MARVELS CCF peak varies with phase for TYC 3010 (red) and another MARVELS candidate, TYC 1275-00027-1 (black). The MARVELS spectrograph does not possess the resolution to resolve two separate peaks in the CCF for TYC 3010, even at periastron. Instead the width of the CCF broadens dramatically, and upon inspection the peak appears asymmetric with a slight "shoulder" that suggests the presence of an unresolved secondary peak. This large variation in the peak width is not observed in TYC 1275-00027-1, which is known to be a single star. Therefore, by monitoring how the CCF peak changes with phase, and through visual inspection of the peaks, surveys can identify systems that are likely to be false positives like TYC 3010 during the candidate-vetting process. Finally, the median value of the CCF peak width is larger for TYC 3010 than the comparison star, but this may be due to either TYC 3010 rotating faster or the presence of the secondary peak. When confronted with a system whose peak is consistently broader than one might expect for a typical solar-type star, further investigation is necessary to determine if it is merely a fast rotator or if it has a stellar companion.

(A color version of this figure is available in the online journal.)

the stars spend a long time ( $\sim 7$ months) away from periastron, which is precisely the moment when the RVs of the components are disparate enough for it to be fairly easy to resolve the two sets of spectral lines, and the large RV amplitude $\left(\sim 15-20 \mathrm{~km} \mathrm{~s}^{-1}\right)$ is indicative of a stellar binary with two solar-type stars. Moreover, the orientation makes it so that only a relatively small component of the orbital velocities is directed along our line of sight. Finally, the cadence of the MARVELS survey made it unlikely to observe multiple epochs of periastron.

\subsection{How RV Surveys Can Identify Astrophysical False Positives like TYC 3010}

For any given RV survey, the lower the resolution of the spectrograph, the more vigilant one must be for these kinds of false positives. For TYC 3010 in particular, a spectrograph with a resolution of $R \gtrsim 50,000$ is required to resolve the spectral lines throughout most of the orbit. But in general, as the resolution (and cadence of observations) decreases, the wider the range of eccentricities, arguments of periastron, and orbital periods by which stellar binaries could masquerade as substellar companions for significant fractions of their orbits.

Furthermore, longer period orbits $(P \gtrsim 1 \mathrm{yr})$ should be handled with special care, for in these cases the phase coverage is more likely to be incomplete. In order to survey $\sim 3000$ stars over four years, MARVELS required a cadence that made it less likely to observe multiple epochs of periastron for a binary with the period of TYC 3010. For MARVELS and similar $\mathrm{RV}$ surveys for substellar companions, it can be costly to use precious resources to examine false positives. Therefore, in this section, we describe a method that the MARVELS team currently employs to identify binaries like TYC 3010 during the candidate-vetting process.
For typical RV surveys today, a standard line bisector analysis can usually be performed to assess the presence of blended double-lined binaries. However, this was not possible for the MARVELS discovery data due to its limited spectral resolution. Thus, following our experience with TYC 3010, MARVELS has developed an internal pipeline for inspecting the widths of the CCF peaks for all of our candidates. This way, we can readily monitor the $\mathrm{CCFs}$ for signs that indicate that there may be more than one stellar component present (e.g., the large excursions in the width of the CCF peak that occur near periastron for TYC 3010; see Figure 11). There are two properties of the CCFs that we now monitor: (1) the average width of the CCF peak compared to other stars in the survey, and (2) any other significant changes in the shape of the CCF over time.

For a typical solar-type star that is not rotating too rapidly (i.e., the kinds of stars that MARVELS targets), one would expect the width of the CCF peak to be $\sim 10 \mathrm{~km} \mathrm{~s}^{-1}$, which is largely the result of thermal broadening and micro-turbulence. However, when binary systems like TYC 3010 are unresolved, the widths of the CCF peak are broader $\left(\sim 20 \mathrm{~km} \mathrm{~s}^{-1}\right)$, indicating that there may be multiple stellar components contributing to the flux from the system (see Figure 11). In fact, an atypically broad CCF peak could also be the result of a single star rotating atypically fast, so a broad peak is not in itself sufficient to identify the system as a binary. Nevertheless, a broad peak should be taken as a sign to proceed with caution. Furthermore, changes in the skewness of the $\mathrm{CCF}$ peak might provide an even more sensitive diagnostic for these kinds of systems. Thus, by monitoring changes in the CCF peak, even if one misses the small fraction of the orbit where, depending on the resolution, the CCF peak either broadens dramatically or separates into distinct peaks (or if one 
is suspicious of the relatively few epochs where the system happened to be caught near periastron), it is possible to flag systems like TYC 3010, which may contain much more mass than most of the RV data suggests.

The case of TYC 3010 is also a pertinent lesson on how important it is to handle outliers carefully, especially in this era of large surveys where thousands of objects must be screened for the most favorable candidates. We possessed plausible reasons for suspecting that the outliers in the discovery data might be spurious (known issues with fiber mis-pluggings; low $\mathrm{S} / \mathrm{N}$; and the outliers were only detected during one of the three orbits observed). Moreover, and perhaps ironically, the spurious orbit solution is actually a better fit to the discovery data (excluding the outliers) than the true orbit solution, because of the need to disentangle the primary and secondary RV components from the (apparently) single-lined RV measurements. However, even when faced with such a compelling initial solution and sensible reasons for considering the outliers to be invalid, it is imperative to investigate further and provide evidence that the reasons for rejecting the outliers are not only plausible but justified.

Furthermore, when the analysis is distributed among multiple team members like it is within MARVELS, it is necessary to make sure each step of the analysis is documented as clearly as possible. For MARVELS, the members who perform the candidate-vetting are usually different from those who perform the subsequent analysis for each candidate, so it is important for each team member to be able to readily discover if any outliers were rejected and why. MARVELS has now modified its internal analysis tracking system in order to make the entire analysis process more transparent.

Finally, if we had been monitoring the widths of the CCF peaks, we could have considered the evidence of the broad peak, as well as the changing peak width around periastron, though in truth neither the changing width nor the broad peak by themselves would have likely been sufficiently compelling to reject the initial orbit solution. In the end, the most important part of our analysis was to strategically focus our HET/HRS observations on periastron, the phase where the outliers occurred and where it was easiest to resolve the spectral lines. This strategy would have been more difficult with a conventionally scheduled telescope, but was readily achieved with the queuescheduled nature of the HET.

\section{SUMMARY}

We have demonstrated, using high resolution spectroscopy, that TYC 3010 is an SB2. We have shown how, with a spectrograph below a given resolution $(R \lesssim 50,000)$, the eccentricity and the orientation of the system with respect to our line of sight allowed a large fraction of the RV curve to appear remarkably similar to the kind of signal one would expect from a BD secondary as opposed to a stellar-mass secondary. Furthermore, as a result of the cadence of the MARVELS survey and the orbital period of the system, we were more likely to miss periastron during a given orbit. Thus, we were more susceptible to rejecting the periastron points we did obtain as outliers, even though these points are where the spectral lines are most widely separated, and thereby where it is easiest to determine that the system is an SB2.

Finally, we concluded with a word of warning to RV surveys, since for a given resolution and cadence, there are a range of orbital parameters that can make a stellar-mass binary companion appear to be substellar. The lower the resolution or cadence, the greater the number of stellar binaries that can masquerade in a fashion similar to TYC 3010. Therefore, if other surveys can carefully monitor the widths of the CCF peaks for their targets (or monitor their line bisectors if they have high enough resolution), and when possible, focus their resources on observations of peripassage, then we hope that they will be able to avoid similar astrophysical false positives.

This research was partially supported by the Vanderbilt Initiative in Data-Intensive Astrophysics (VIDA) and NSF CAREER grant AST 0349075 (C.E.M., K.G.S., L.H., J.P.), NSF AAPF AST 08-02230 (J.P.W.), NSF CAREER grant AST 0645416 (E.A.), CNPq grant 476909/2006-6 (G.F.P.M.), FAPERJ grant APQ1/26/170.687/2004 (G.F.P.M.), NSF CAREER grant AST-1056524 (B.S.G., J.D.E.), and a PAPDRJ CAPES/FAPERJ Fellowship (L.G.).

Based on observations with the SDSS $2.5 \mathrm{~m}$ telescope. Funding for the MARVELS multi-object Doppler instrument was provided by the W. M. Keck Foundation and NSF grant AST-0705139. The MARVELS survey was partially funded by the SDSS-III consortium, NSF grant AST-0705139, NASA with grant NNX07AP14G and the University of Florida. The Center for Exoplanets and Habitable Worlds is supported by the Pennsylvania State University, the Eberly College of Science, and the Pennsylvania Space Grant Consortium.

Data presented herein were obtained at the Hobby-Eberly Telescope (HET), a joint project of the University of Texas at Austin, the Pennsylvania State University, Stanford University, Ludwig-Maximilians-Universität München, and Georg-AugustUniversität Göttingen. The HET is named in honor of its principal benefactors, William P. Hobby and Robert E. Eberly.

This research has made use of the SIMBAD database, operated at CDS, Strasbourg, France, and the AAVSO Photometric All-Sky Survey (APASS), funded by the Robert Martin Ayers Sciences Fund. It also made use of the IRAF software distributed by the National Optical Astronomy Observatory, which is operated by the Association of Universities for Research in Astronomy (AURA) under cooperative agreement with the National Science Foundation. This publication makes use of data products from the Two Micron All Sky Survey, which is a joint project of the University of Massachusetts and the Infrared Processing and Analysis Center/California Institute of Technology, funded by the National Aeronautics and Space Administration and the National Science Foundation. We also make use of data products from the Wide-field Infrared Survey Explorer, which is a joint project of the University of California, Los Angeles, and the Jet Propulsion Laboratory/California Institute of Technology, funded by the National Aeronautics and Space Administration. Funding for SDSS-III has been provided by the Alfred P. Sloan Foundation, the Participating Institutions, the National Science Foundation, and the U.S. Department of Energy Office of Science. The SDSS-III Web site is http://www.sdss3.org/.

SDSS-III is managed by the Astrophysical Research Consortium for the Participating Institutions of the SDSS-III Collaboration including the University of Arizona, the Brazilian Participation Group, Brookhaven National Laboratory, University of Cambridge, University of Florida, the French Participation Group, the German Participation Group, the Instituto de Astrofisica de Canarias, the Michigan State/Notre Dame/ JINA Participation Group, Johns Hopkins University, Lawrence Berkeley National Laboratory, Max Planck Institute for Astrophysics, New Mexico State University, New York University, 
Ohio State University, Pennsylvania State University, University of Portsmouth, Princeton University, the Spanish Participation Group, University of Tokyo, University of Utah, Vanderbilt University, University of Virginia, University of Washington, and Yale University.

Facilities: Sloan (MARVELS), ARC (ARCES), HET (HRS), Keck:I (NIRC2), Sanchez (FastCam)

\section{REFERENCES}

Allende Prieto, C., Barklem, P. S., Lambert, D. L., \& Cunha, K. 2004, A\&A, 420, 183

Allende Prieto, C., \& Lambert, D. L. 2000, AJ, 119, 2445

Baranne, A., Queloz, D., Mayor, M., et al. 1996, A\&AS, 119, 373

Bender, C. F., Mahadevan, S., Deshpande, R., et al. 2012, ApJL, 751, L31

Bertelli, G., Bressan, A., Chiosi, C., Fagotto, F., \& Nasi, E. 1994, A\&AS, 106,275

Bouchy, F., \& SOPHIE Team. 2006, in Tenth Anniversary of 51 Peg-b: Status of and Prospects for Hot Jupiter Studies, ed. L. Arnold, F. Bouchy, \& C. Moutou, 319

Castelli, F., \& Kurucz, R. L. 2004, arXiv:astro-ph/0405087

Crifo, F., Jasniewicz, G., Soubiran, C., et al. 2010, A\&A, 524, A10

Cutri, R. M., Skrutskie, M. F., van Dyk, S., et al. 2003, yCat, 2246, 0

De Lee, N., Ge, J., Crepp, J., et al. 2013, AJ, submitted

Delfosse, X., Forveille, T., Ségransan, D., et al. 2000, A\&A, 364, 217

Demarque, P., Woo, J.-H., Kim, Y.-C., \& Yi, S. K. 2004, ApJS, 155, 667

Duquennoy, A., \& Mayor, M. 1991, A\&A, 248, 485

Eastman, J., Gaudi, B. S., \& Agol, E. 2013, PASP, 125, 83

Eisenstein, D. J., Weinberg, D. H., Agol, E., et al. 2011, AJ, 142, 72

Erskine, D. J. 2003, PASP, 115, 255

Femenía, B., Rebolo, R., Pérez-Prieto, J. A., et al. 2011, MNRAS, 413, 1524

Fleming, S. W., Ge, J., Barnes, R., et al. 2012, AJ, 144, 72

Fleming, S. W., Ge, J., Mahadevan, S., et al. 2010, ApJ, 718, 1186

Ge, J. 2002, ApJL, 571, L165

Ge, J., \& Eisenstein, D. 2009, astro2010: The Astronomy and Astrophysics Decadal Survey, arXiv:0903.0181

Ge, J., Erskine, D. J., \& Rushford, M. 2002, PASP, 114, 1016

Ge, J., Lee, B., de Lee, N., et al. 2009, Proc. SPIE, 7440, 74400L

Ge, J., Mahadevan, S., Lee, B., et al. 2008, in ASP Conf. Ser. 398, Extreme Solar Systems, ed. D. Fischer, F. A. Rasio, S. E. Thorsett, \& A. Wolszczan (San Francisco, CA: ASP), 449

Ge, J., van Eyken, J., Mahadevan, S., et al. 2006, ApJ, 648, 683

Girardi, L., Bertelli, G., Bressan, A., et al. 2002, A\&A, 391, 195

González Hernández, J. I., Bonifacio, P., Ludwig, H.-G., et al. 2008, A\&A, 480, 233

Gudehus, D. H. 2001, BAAS, 33, 850

Gunn, J. E., Siegmund, W. A., Mannery, E. J., et al. 2006, AJ, 131, 2332

Hauschildt, P. H., Allard, F., \& Baron, E. 1999, ApJ, 512, 377

Henden, A. A., Levine, S. E., Terrell, D., Smith, T. C., \& Welch, D. 2012 JAAVSO, 40, 430
Henry, T. J. 2004, in ASP Conf. Ser. 318, Spectroscopically and Spatially Resolving the Components of the Close Binary Stars, ed. R. W. Hilditch, H. Hensberge, \& K. Pavlovski (San Francisco, CA: ASP), 159

Henry, T. J., Franz, O. G., Wasserman, L. H., et al. 1999, ApJ, 512, 864

Høg, E., Fabricius, C., Makarov, V. V., et al. 2000, A\&A, 355, L27

Jiang, P., Ge, J., Cargile, P., et al. 2013, AJ, submitted

Kurucz, R. 1993, ATLAS9 Stellar Atmosphere Programs and 2 km/s Grid. Kurucz CD-ROM No. 13 (Cambridge, MA: Smithsonian Astrophysical Observatory), 13

Lee, B. L., Ge, J., Fleming, S. W., et al. 2011, ApJ, 728, 32

Lytle, D. M. 1993, in ASP Conf. Ser. 52, Astronomical Data Analysis Software and Systems II, ed. R. J. Hanisch, R. J. V. Brissenden, \& J. Barnes (San Francisco, CA: ASP), 18

Ma, B., Ge, J., Barnes, R., et al. 2013, AJ, 145, 20

Mamajek, E. 2011, Univ. Rochester Internal Memorandum, http://www.pas. rochester.edu/ emamajek/EEM_dwarf_UBVIJHK_color_Teff.dat

Mandushev, G., Torres, G., Latham, D. W., et al. 2005, ApJ, 621, 1061

Marcy, G. W., \& Butler, R. P. 2000, PASP, 112, 137

Matthews, K., \& Soifer, B. T. 1994, in Astronomy with Arrays: The Next Generation, ed. I. S. McLean (Astrophysics and Space Science Library, Vol. 190; Berlin: Springer), 239

Nidever, D. L., Marcy, G. W., Butler, R. P., Fischer, D. A., \& Vogt, S. S. 2002, ApJS, 141, 503

Oscoz, A., Rebolo, R., López, R., et al. 2008, Proc. SPIE, 7014, 701447

Pepe, F., Mayor, M., Delabre, B., et al. 2000, Proc. SPIE, 4008, 582

Queloz, D., Mayor, M., Weber, L., et al. 2000, A\&A, 354, 99

Raghavan, D., McAlister, H. A., Henry, T. J., et al. 2010, ApJS, 190, 1

Ramírez, I., Allende Prieto, C., \& Lambert, D. L. 2007, A\&A, 465, 271

Ramsey, L. W., Adams, M. T., Barnes, T. G., et al. 1998, Proc. SPIE, 3352, 34

Reddy, B. E., Lambert, D. L., \& Allende Prieto, C. 2006, MNRAS, 367, 1329

Schlegel, D. J., Finkbeiner, D. P., \& Davis, M. 1998, ApJ, 500, 525

Shetrone, M., Cornell, M. E., Fowler, J. R., et al. 2007, PASP, 119, 556

Sneden, C. A. 1973, PhD thesis, The University of Texas at Austin

Sousa, S. G., Santos, N. C., Israelian, G., Mayor, M., \& Monteiro, M. J. P. F. G. 2007, A\&A, 469, 783

Sousa, S. G., Santos, N. C., Mayor, M., et al. 2008, A\&A, 487, 373

Tabernero, H. M., Montes, D., \& González Hernández, J. I. 2012, A\&A, 547, A13

Torres, G., Andersen, J., \& Giménez, A. 2010, A\&ARv, 18, 67

Tull, R. G. 1998, Proc. SPIE, 3355, 387

van Eyken, J. C., Ge, J., \& Mahadevan, S. 2010, ApJS, 189, 156

Wang, J., Ge, J., Jiang, P., \& Zhao, B. 2011, ApJ, 738, 132

Wang, J., Ge, J., Wan, X., De Lee, N., \& Lee, B. 2012a, PASP, 124, 1159

Wang, J., Ge, J., Wan, X., Lee, B., \& De Lee, N. 2012b, PASP, 124, 598

Wang, S.-i., Hildebrand, R. H., Hobbs, L. M., et al. 2003, Proc. SPIE, 4841, 1145

Wisniewski, J. P., Ge, J., Crepp, J. R., et al. 2012, AJ, 143, 107

Wright, E. L., Eisenhardt, P. R. M., Mainzer, A. K., et al. 2010, AJ, 140, 1868

Wright, J., Roy, A., Mahadevan, S., et al. 2013, ApJ, submitted

Xia, F., \& Fu, Y.-N. 2010, ChA\&A, 34, 277

Xia, F., Ren, S., \& Fu, Y. 2008, Ap\&SS, 314, 51

Zucker, S. 2003, MNRAS, 342, 1291

Zucker, S., \& Mazeh, T. 1994, ApJ, 420, 806 